\title{
Harvesting a remote renewable resource
}

\author{
Thorsten Upmann ${ }^{1,2,3}$ (1) . Stefan Behringer ${ }^{4}$
}

Received: 14 June 2019 / Accepted: 20 February 2020

(c) The Author(s) 2020

\begin{abstract}
In standard models of spatial harvesting, a resource is distributed over a continuous domain with an agent who may harvest everywhere all the time. For some cases though (e.g., fruits, mushrooms, algae), it is more realistic to assume that the resource is located at a fixed point within that domain so that an agent has to travel in order to be able to harvest. This creates a combined travelling-and-harvesting problem where slower travel implies a lower travelling cost and, due to a later arrival, a higher abundance of the resource at the beginning of the harvesting period; this, though, has to be traded off against less time left for harvesting, given a fixed planning horizon. Possible bounds on the controls render the problem even more intricate. We scrutinise this bioeconomic setting using a two-stage optimal control approach, and find that the agent economises on the travelling cost and thus avoids to arrive at the location of the resource too early. More specifically, the agent adjusts the travelling time so as to be able to harvest with maximum intensity at the beginning and the end of the harvesting period, but may also find it optimal to harvest at a sustainable level, where the harvesting and the growth rate of the stock coincide, in an intermediate time interval.
\end{abstract}

Keywords Optimal travelling-and-harvesting decision · Spatial renewable resource · Bioeconomic model $\cdot$ Two-stage optimal control problem · Sustainable harvesting

\section{Introduction}

The spatial dimension has recently attracted substantial attention in the economic literature on management of renewable resources. Frequently, this literature focuses on how much effort is required to harvest a resource when that resource is moving (e.g., fish or game). ${ }^{1}$ In this paper, we

\footnotetext{
${ }^{1}$ See, for example, Neubert (2003), Neubert and Herrera (2008), Brock and Xepapadeas (2008), Brock and Xepapadeas (2010), Ding and Lenhart (2009), Moeller and Neubert (2013), Kelly et al. (2016), and Grass et al. (2019).
}

Thorsten Upmann

Thorsten.Upmann@hifmb.de

Stefan Behringer

Stefan.Behringer@sciencespo.fr

1 Helmholtz-Institute for Functional Marine Biodiversity at the University of Oldenburg (HIFMB), Ammerländer Heerstraße 231, 23129 Oldenburg, Germany

2 Bielefeld University, Bielefeld, Germany

3 CESifo, Munich, Germany

4 Sciences Po, Department of Economics, 28 rue des Saints Pères, 75007 Paris, France reverse this premise: we consider the case where an agent is required to move in order to harvest an immobile resource. Since travelling is a pre-requisite of harvesting, this implies a spatio-temporal interdependence of both policies, which is the focus of this paper.

The management of renewable natural resources has been a central issue in economics for many decades, and meanwhile the early models have been extended and generalised in various respects. ${ }^{2}$ While the temporal dimension of bioeconomic problems has been considered already in the early works, the spatial dimension became of interest to economists rather late, although it had been treated in the theoretical biology and applied mathematics literature for some time. Only in 1999, Sanchirico and Wilen generalise the fundamental open-access models of Gordon (1954) and Smith (1968). They set up a bioeconomic model with a finite number of resource patches within which the resource migrates, resulting in time-dependent changes in

\footnotetext{
${ }^{2}$ For example, Fan and Wang (1998) generalise the optimal harvesting policy of an autonomous harvesting problem with logistic growth to a non-autonomous case with periodic coefficients; Liski et al. (2001), accounting for costly changes of the harvesting rate, explore the effects of increasing returns to scale for a standard fishery management model; and Feichtinger et al. (2003), Hritonenko and Yatsenko (2006), Tahvonen (2008, 2009a, b), Skonhoft et al. (2012), Tahvonen et al. (2013) and Belyakov and Veliov (2014) investigate harvesting of age-structured populations.
} 
the allocation of harvesting effort between these patches. In this way, Sanchirico and Wilen (1999) integrate withinand between-patch biological and economic forces and demonstrate how these effects determine the process of bioeconomic convergence over space and time.

Following Sanchirico and Wilen (1999), the early models in spatial resource economics feature discrete patches, where migration of the biomass is modelled as entry and exit of the biomass from one location to the other. An alternative approach assumes a continuous distribution of the resource and models the migration and the spread of the biomass as diffusion; notable contributions are, for example, Montero (2000, 2001), Neubert (2003), Brock and Xepapadeas (2008, 2010), Ding and Lenhart (2009), Grass et al. (2019). An overview of the literature of spatialdynamic systems in resource economics is provided by Conrad and Smith (2012) and Kroetz and Sanchirico (2015), for example.

In both strands of the literature, it is the resource that is mobile, while a possible movement of the agent remains unrecognised. In many instances, an immobile agent represents a reasonable simplification, as the effect of the agent's movement can be neglected without losing much realism (e.g., coastal fixed-net fishery or shooting game)—but in other cases it is not. For example, in fruit and mushroom harvesting (in distant, vast wilderness such as Canada or Kamchatka), in forestry and in extensive agriculture, it is the agent who is moving to a resource that is positioned at a fixed location. In these cases, the travelling costs are often significant due to either large distances or because of a lack of infrastructure. Moreover, there is frequently only a fixed time slot in which the resource can be harvested, imposing constraints on the arrival time; for example, grape harvesting needs to be done within the last few days before the first night frost (late harvest). Another example, which has recently attracted much attention and for which travelling is essential, is algae: a rapidly growing renewable resource, which may be used in the production of human and animal food, cosmetics, pharmaceuticals, chemicals, plastics, and biofuel. ${ }^{3}$ Algae is frequently located at distant patches and is hard to monitor; in particular, algae farms have now often been automated and located more and more off-shore so that travelling times become increasingly relevant.

Few papers consider a travelling-and-harvesting problem of an agent in a spatial domain. Notable examples are Robinson et al. (2008), Behringer and Upmann (2014), Sirén and Parvinen (2015), Belyakov et al. $(2015,2017)$ and Zelikin et al. (2017), who consider an immobile resource

\footnotetext{
${ }^{3}$ For the possibilities of algal biofuel production, see, for example, Shurin et al. (2013), Zhu et al. (2017), Ummalyma et al. (2017), and Chu (2017); for a paper investigating algae growth control from a pollution point of view, see Yoshioka and Yaegashi (2018).
}

positioned at known locations. Except for Robinson et al. (2008) and Sirén and Parvinen (2015), these authors analyse a resource that is continuously distributed on the periphery of a circle and an agent who leaves for a round trip, returning home after each turn. In those models, the agent does not need to stop in order to harvest the resource, but is able to do this en passant. While in the model of Behringer and Upmann (2014) the harvesting activity does not cost any time over and above the time of travelling, in Belyakov et al. $(2015,2017)$ the maximal harvesting activity is inversely proportional to velocity. Models of the latter type are sometimes referred to as search models, because a higher speed of travelling renders the agent incapable of descrying much of the resource (see also Robinson et al. 2002). However, in both types of models, i.e., in Behringer and Upmann (2014) and in Belyakov et al. $(2015,2017)$, the travelling and the harvesting activity occur simultaneously.

Complementary to that approach are sequential travelling-and-harvesting decisions, which have previously been considered by Robinson et al. (2008) and Sirén and Parvinen (2015), for example. In Robinson et al. (2008), the resource is located at discrete patches, and the agent is required to travel to those locations in order to be able to harvest. Sirén and Parvinen (2015) modify that model and formulate a continuous-time, continuous-space version. In those models, travelling and harvesting are mutually exclusive activities, taking place at different locations at subsequent times. Yet, in both models the decisions about travelling and harvesting are connected via a gatheringspecific cost function. This formalises the idea that the travelling time, and hence the travelling cost, increases in the total amount already harvested, assuming that the harvest has to be carried back to a local market.

In Sirén and Parvinen (2015), speed is assumed to be fixed (thereby invalidating their peculiar assumption that travelling costs are decreasing in speed). As a consequence, travelling costs are linearly increasing in the travelling distance, so that it is the distance, rather than the speed, on which the agent has to decide. In this way, travelling and harvesting decisions collapse, similar to search models. Sirén and Parvinen (2015), considering a infinite time horizon, show that in the steady state, the stock is an increasing function of the distance, while also the harvest increases with distance, before it eventually drops to zero.

In these spatio-temporal harvesting models, travelling to the resource constitutes a crucial activity. In particular, in their numerical analysis Robinson et al. (2008) show that the travelling costs dissipate a substantial fraction of the harvesting yield, and thus of the resource value. Because of this significance of the travelling cost, they obtain cyclical solutions with extraction varying over space and time, where the number of periods is sensitive with respect to parameterisation of the model. 
The prediction of cyclical solutions with extraction varying over space and time, developed by Robinson et al. (2008), resemble those in the continuous time model of Belyakov et al. (2015). These authors, specifying a longrun average objective to motivate a sustainability goal, show the existence of an optimal solution that also reveals a periodic structure with possibly exhausted areas appearing on the periphery of a circle. However, the presence of cyclical solutions is critically dependent on the assumption of harvesting capacity being inversely related to speed, as it is assumed in search models. Behringer and Upmann (2014) and Zelikin et al. (2017) relax this assumption and allow for two fully independent controls for speed and harvesting. Disentangling speed and harvesting, the latter authors obtain non-cyclic, and in some special cases even constant solutions.

In this paper, we also "disentangle" speed and harvesting, but with respect to the time dimension. We consider a twostage optimal control problem with travelling and harvesting taking place in subsequent periods. In this way, travelling and harvesting are independent in the sense that they are only connected by the arrival time of the agent at the location of the resource. At the same time, travelling and harvesting are mutually exclusive, rival activities: the more time is spent on travelling, the less time is left for harvesting, and vice versa. The link by the arrival time yields a truly dynamic two-stage optimal control problem with two interconnected sub-problems: a travelling problem and a subsequent harvesting problem.

Our model shares some similarities with the timber gathering model of Robinson et al. (2008) and, in particular, with the continuous-time, continuous-space model of Sirén and Parvinen (2015). In all three models, travelling and harvesting take place at different locations at subsequent times. However, in our model there neither is a functional link between the cost of travelling and the cost of harvesting, nor is there the need to carry the harvest back to some home market, but the resource is immediately sold at a market (or to a distributor) that is located close to the resource. (For a model that investigates the effects of endogenous market demands in a spatial harvesting setting see Aniţa et al. 2019.) In the absence of such nearby markets, Robinson et al. (2008) allow for low levels of resource density to appear far from the harvester's home with abundant resources in between, generalising the monotone predictions of Sirén and Parvinen (2015). Similarly, Belyakov et al. (2015), assuming an infinite time horizon, allow for a periodic structure with possibly exhausted areas. In our model, though, we investigate the behaviour of an agent with a finite rather than an infinite planning horizon. This perspective necessarily constrains the time the agent may employ for harvesting once the resource is reached, rendering the eventual resource pattern a function of temporal and transport constraints.

To specify our two-stage travelling-and-harvesting control problem, we assume a linear-quadratic travelling cost, and consider two different possible specifications for the growth process of the resource: exponential growth and logistic growth. Applying these specifications, we analytically derive the optimal travelling-and-harvesting policies, i.e., the optimal paths, and the associated profits for both types of growth functions, thereby demonstrating the interdependence between both problems. The need for travelling requires the agent to weigh the benefit of an early arrival, i.e., a longer harvesting period, with its cost: a higher pecuniary travelling cost and a shorter time for the resource to grow, implying a lower stock upon arrival and hence less beneficial conditions for harvesting. In essence, the limited amount of time (fixed time period) encourages the agent to start harvesting early, while the presence of travelling costs lets the agent postpone the beginning of the harvesting period.

More specifically, we show that the optimal policies for exponential and logistic growth feature similar characteristics depending on two fundamental parameters: the planning horizon and the harvesting capacity (maximal effort level). Given those parameters, the travelling period provides the agent with the possibility to customise the length of the harvesting period by choosing the arrival time. Since early arrival is costly in terms of higher travelling cost, the agent adjusts the speed of travelling to avoid being idle. As a consequence, optimal harvesting is done either at the maximal rate all the time, or harvesting is reduced to a sustainable level during an intermediate time interval. The latter policy with two switches in the harvesting intensity (maximumsustainable-maximum) only occurs in case of the logistic growth function. Finally, we also investigate the sensitivity of our results with respect to the presence of bounds on the control of movement and with repect to the rate at which future revenues and costs are discounted. We show that, even though these modifications lead to some shifts in the optimal policy, the basic effects still prevail.

The rest of the paper is structured as follows: In Section "The model" we set up the model. In Section "Decomposition of the problem" we decompose the travelling-and-harvesting problem into the two sub-problems. We begin our analysis with the harvesting problem in Section "Second stage: harvesting", and we then proceed with an analysis of the full travelling-and-harvesting problem in Section "First stage: optimal travelling-and-harvesting policy". In Section "Robustness of the results" we discuss the robustness of our results with respect to bounds on the control and the 
discount rate, before we conclude in Section "Conclusion". The robustness analysis is relegated to Appendix A; longer proofs, to Appendix B.

\section{The model}

We consider an economic agent who has the exclusive right to harvest a renewable natural resource during a fixed, finite time period $\mathscr{T} \equiv[0, T]$. We may think of a harvesting licence or a rental period that begins at time $t=0$ and terminates at $t=T{ }^{4}$ The resource is situated at some fixed and known location $x_{1} \in(0, \bar{x}]$. At time $t \in \mathscr{T}$ the location of the economic agent is $x(t) \in \mathscr{X} \equiv[0, \bar{x}]$, with the agent's initial location given by $x(0)=0$. The agent is able to harvest the resource only at his/her current position, and since $x(0) \neq x_{1}$, the agent is required to travel to get access to the resource. Only on arrival at location $x_{1}$ is the agent able to begin with harvesting. The agent's problem is thus a combined travelling-and-harvesting problem, where the speed of travelling, and hence the arrival time, and the harvesting rate have to be determined jointly in order to maximise the total profit, composed of the revenue from harvesting net of harvesting and travelling costs.

In order to move from one location to the next, the agent has to adjust the velocity of travelling $v(t) \in \mathscr{V} \subset \mathbb{R}$. This speed of movement (or the harvesting machine) cannot be chosen directly, though, but is physically controlled by means of acceleration $a(t) \in \mathscr{A} \subset \mathbb{R}$. Thus, movement is described by

$\dot{x}(t)=v(t), \quad \dot{v}(t)=a(t), \quad \forall t \in \mathscr{T}$ with $x(0)=v(0)=0$.

Specifying acceleration, rather than speed, as the control variable avoids the occurrence of unrealistic, i.e., discontinuous speed profiles where the agent may abruptly switch speeds. (This specification of the movement model follows Pontryagin et al. 1962; Léonard and Long 1992, Sec. 8.1; Hull 2003, Sec. 17.7 and others; a more sophisticated model can be found in, e.g., Bertolazzi and Frego 2018.) There may be lower and upper bounds on acceleration. For the moment, we disregard such constraints, but we shall assume in Appendix A, that acceleration is bounded by $a(t) \in \mathscr{A} \equiv$ $[\underline{a}, \bar{a}]$ with $\underline{a}<0$ and $\bar{a}>0 .^{5}$

\footnotetext{
${ }^{4}$ With an infinite time horizon the significance of the travelling period will reduce and the trade-off between the travelling and the harvesting decision will be less pronounced; also, any end-of-period effects will vanish.-All of those effects would distract from the central issue of this paper.

${ }^{5}$ The minimum acceleration $\underline{a}$ is necessarily negative to allow for a slowdown of speed, as the agent would otherwise be unable to stop-and start harvesting.
}

Since both harvesting and travelling take time and the time horizon is finite, the earlier the agent arrives at location $x_{1}$ the more time is left for harvesting. In order to render the problem non-trivial, we subsequently assume that the costs of travelling are not too high, so that an arrival before time $T$ is desirable. Since speed is finite, the arrival time must be strictly positive. Formally, the arrival time of the agent at the location of the resource $x_{1}$ is the first time the agent's location is $x_{1}$ :

$t_{1} \equiv \min _{t}\left\{t \in \mathscr{T} \mid x(t)=x_{1}, v(t)=0\right\}$.

Then, $\Lambda \equiv\left[0, t_{1}\right]$ denotes the agent's travelling period; and $\Delta \equiv\left(t_{1}, T\right]$, the resulting harvesting period. ${ }^{6}$ The total time available is then spent on travelling and on harvesting, with the travelling period preceding the harvesting period. This is illustrated in Fig. 1.

The stock of the renewable resource (i.e., the biomass) at time $t \in \mathscr{T}$ is denoted by $s(t) \geq 0$. We assume that the resource is growing at rate $g(s)$ with $g(0)=0$. (Subsequently, we will consider the cases of exponential and logistic growth.) Furthermore, harvesting gradually diminishes the stock. The harvest depends on the abundance of the resource, i.e., on the stock $s$, and on the harvesting effort $h(t) \in \mathscr{H} \equiv[0, \bar{h}] \subset \mathbb{R}_{+}$. Suppose that for a given stock, the yield from harvesting increases in effort; moreover, effort is more productive the higher the stock. Specifically, we assume that the harvesting yield equals zero, $H=0$, if either the stock or the effort equals zero, i.e., if $s=0$ or $h=0$. To capture this idea, we follow the familiar Schaefer model (see Schaefer 1954) and specify the revenue from harvesting as a bilinear function of effort and the stock: $H(t)=q s(t) h(t)$, where $q$ is the catchability coefficient, defined as the fraction of the stock harvested per unit of effort. For convenience, we normalise the units of effort and set $q=1$. Then, the harvesting yield equals $H(t)=h(t) s(t)$ if the agent's location is $x_{1}$, and $H(t)=0$ otherwise. With this specification, the harvesting effort equally represents the harvesting rate, and we thus use the phrases harvesting effort and harvesting rate interchangeably. ${ }^{7}$ Putting pieces together, the resulting growth of the stock is governed by the differential equation

$\dot{s}(t)=g(s(t))-h(t) s(t) \mathbf{1}_{\Delta}(t), \quad \forall t \in \mathscr{T}, s(0)=s_{0} .(1 \mathrm{c})$

\footnotetext{
${ }^{6}$ Once the agent has reached location $x_{1}$, they will never start travelling again, and thus the agent completes the planning period at location $x_{1}$, i.e., $x(t)=x_{1}, \forall t \in\left[t_{1}, T\right]$.

${ }^{7}$ The assumption that the agent chooses the harvesting rate $h=\mathrm{H} / \mathrm{s}$ is justified by the idea of stock-dependent harvesting effort, where the yield from a given amount of effort depends on the abundance of the resource; harvesting effort is then similar to fishing by nets, which captures a fraction of the fish stock. If we departed from that view and instead assumed that the agent may choose the harvesting amount $H$ directly, this would affect the dynamics of the optimally controlled system. We are very grateful for a referee for pointing this out.
} 


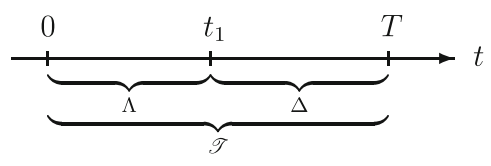

Fig. 1 Structure of the planning period $\mathscr{T}$ : the travelling period $\Lambda$ ends when the harvesting period starts at the arrival time $t_{1}$

where $\mathbf{1}_{\Delta}$ denotes the indicator function for the harvesting period, i.e., for times $t \in \Delta$.

Travelling and harvesting are both costly. We assume that the harvesting cost $C(H)$ is increasing and convex, i.e., $C^{\prime}>0$ and $C^{\prime \prime} \geq 0$ for all $H \in \mathbb{R}_{+}$, with $C(0)=0$. More specifically, we assume that the harvesting cost is linear in total catch, $C(H)=c H=c h s$, with $0 \leq c<p$. Then, instantaneous profit from harvesting amounts to $(p-c) h(t) s(t)$ or, more compactly, $M h(t) s(t)$, where $M \equiv$ $p-c$ denotes the per-unit profit (mark-up). Also, travelling is associated with some cost, which generically depends on both speed and acceleration: $K(v, a)$ for $v \in \mathscr{V}$ and $a \in \mathscr{A}$. We assume that pausing is costless, $K(0,0)=0$, that the travelling cost increases with both speed and acceleration, and that acceleration is more costly the higher the speed, i.e., the partial derivatives of $K$ satisfy $K_{v} \geq 0, K_{a} \geq 0$ and $K_{v a} \geq 0$.

Let $\rho \geq 0$ denote the discount rate of the agent, and let $p$ be the (constant) price of one unit of the harvested resource. The problem for the agent is then to maximise the discounted profit flow consisting of instantaneous revenue net of the harvesting cost and net of the travelling cost for the planning period $\mathscr{T} .{ }^{8}$ Presupposing that the agent reasonably chooses $h(t)=0, \forall t \in \Lambda$ and $a(t)=0, \forall t \in \Delta$ (the choices $h(t)>0$ for $t \in \Lambda$ or $a(t)>0$ for $t \in \Delta$ would be futile actions), we obtain the travelling cost

$J_{1}\left(a(\cdot), t_{1}\right) \equiv \int_{0}^{t_{1}} e^{-\rho t} K(v(t), a(t)) \mathrm{d} t$

and the profit from harvesting

$J_{2}\left(h(\cdot), t_{1}\right) \equiv \int_{t_{1}}^{T} e^{-\rho t} M h(t) s(t) \mathrm{d} t$,

where $v$ and $x$, and hence the arrival time $t_{1}$, depend on the acceleration path $a(\cdot)$. We henceforth write, with minor sloppiness, $a(\cdot) \in \mathscr{A}$ and $h(\cdot) \in \mathscr{H}$ as shorthand notations for the admissible paths $(a(t))_{t \in \Lambda}, a(t) \in \mathscr{A}$ and $(h(t))_{t \in \Delta}, h(t) \in \mathscr{H}$, respectively. Putting the pieces together, let $J\left(a(\cdot), h(\cdot), t_{1}\right) \equiv-J_{1}\left(a(\cdot), t_{1}\right)+J_{2}\left(h(\cdot), t_{1}\right)$,

\footnotetext{
${ }^{8}$ In order to simplify the presentation and to focus on the link between the travelling and the harvesting period, we disregard any residual value $\phi(s(T), T)$ that the agent may receive at the end of the planning period. Still, one can easily take into account a residual value, as the only effect of $\phi$ is to modify the transversality condition of the costate variable (see, e.g., Léonard and Long 1992, Theorem 7.2.1).
}

then the agent's optimisation problem reads as

$$
\begin{array}{r}
\max _{\left\{a(\cdot) \in \mathscr{A}, h(\cdot) \in \mathscr{H}, t_{1} \in \mathscr{T}\right\}} J\left(a(\cdot), h(\cdot), t_{1}\right) \\
\text { s.t. }(1 \mathrm{a}),(1 \mathrm{~b}),(1 \mathrm{c}), v(t) \in \mathscr{V} .
\end{array}
$$

The state constraint $s(T) \geq 0$ is automatically fulfilled due to assumptions $g(0)=0$, and $H=0$ whenever $s=0$; similarly, the constraints $x(t)=x_{1}$ and $v(t)=0, \forall t \in \Delta$ are implied by Eq. (1a) and (1c). Thus, those constraints need not be specified explicitly in Eq. (1d).

\section{Decomposition of the problem}

In order to solve problem (1), we draw upon the literature of two-stage optimal control problems, notably on the work of Amit (1986), Tomiyama (1985) and Tomiyama and Rossana (1989), ${ }^{9}$ and decompose the intertemporal optimal travelling-and-harvesting problem into two subsequent problems: a travelling and a harvesting sub-problem. In the travelling problem we choose an acceleration path $a(\cdot)$, and thus the arrival time $t_{1}$, so as to move from location 0 to location $x_{1}$ at minimal cost:

$\min _{\left\{a(\cdot) \in \mathscr{A}, t_{1} \in \mathscr{T}\right\}} J_{1}\left(a(\cdot), t_{1}\right) \quad$ s.t. $\quad(1 \mathrm{a}),(1 \mathrm{~b})$.

Since the travelling time $t_{1}$ can be chosen subject to the constraint $x\left(t_{1}\right)=x_{1}$, we face a free-terminal-time problem with a fixed endpoint constraint.

During the travelling period $\Lambda$, the resource grows unimpaired until the agent arrives at the location $x_{1}$ at time $t_{1}$, when the harvesting period begins. As a consequence, the stock of the resource at the time of arrival, $s\left(t_{1}\right)$, represents the solution of the (interim) growth process $\dot{s}(t)=g(s(t))$ with $s(0)=s_{0}$ for all $t \in \Lambda$. In this way, the travelling decision determines the initial value of the stock process for the harvesting problem, $s_{1}=s\left(t_{1}\right)$. Given those parameters, the resulting subsequent harvesting problem becomes choosing a path of the harvesting effort $h(\cdot)$ that maximises $J_{2}$ :

$$
\max _{\{h(\cdot) \in \mathscr{H}\}} J_{2}\left(h(\cdot), t_{1}\right) \quad \text { s.t. } \quad(1 \mathrm{c}), s(T) \text { free. }
$$

The fact that the travelling time of the agent also represents the growth time of the resource is the crucial link between the travelling problem (2a) and the harvesting problem (2b). As a consequence, the agent has to take into account

\footnotetext{
${ }^{9}$ These authors provide optimality conditions for two-stage, finite time dynamic optimization problems. An extension to an infinite horizon is provided by Makris (2001); and applications of this theory to two-stage optimal control problems can, for example, be found in Boucekkine et al. (2004), Saglam (2011), Grass et al. (2012), Caulkins et al. (2013), Krawczyk and Serea (2013), Moser et al. (2014), Long et al. (2017), and Seidl et al. (2018).
} 
that a longer travelling time reduces the time left for harvesting, and thus ceteris paribus the resulting yield. In contrast, a lower speed of travelling makes travelling less expensive and gives the resource more time to grow, thus providing the opportunity for a more abundant harvest at later times. The travelling-and-harvesting problem (1) takes into account these interdependencies between subproblems (2a) and (2b).

We next derive necessary conditions for an optimal control pair $\left(a^{*}(\cdot), h^{*}(\cdot), t_{1}^{*}\right)$, or for short $\left(a^{*}, h^{*}, t_{1}^{*}\right)$, by decomposing the original problem (1), i.e., the sequence of interdependent problems, into two standard problems. We first consider the harvesting problem of the second stage (2b), and then the travelling problem of the first stage (2a), acknowledging that the solution of the second stage depends on the decision in the first stage. Formally, we proceed as follows: Assuming the existence of the optimal switching time $t_{1}$ in the interior of the time interval $\mathscr{T}$, we solve the second-stage problem and calculate the maximised objective function $J_{2}^{*}$ as a function of the initial state $s_{1}$ and the switching time $t_{1}$. Then, we derive the optimal control $a^{*}$ and the associated optimal switching time $t_{1}$ by solving the travelling problem of the first stage.

\section{Second stage}

Given the control time interval $\Delta$ and the initial condition $s\left(t_{1}\right)=s_{1}$, we solve problem (2b) for an admissible optimal control $h^{*}$. This problem is of a standard form and can be solved using the well-known Pontryagin maximum principle (see, for example, Kamien and Schwartz 1991). Using the solution of the second-stage problem, $\left(h^{*}, s^{*}\right)$, which depends on the starting values $s_{1}$ and $t_{1}$, we calculate the maximised objective value $J_{2}^{*}\left(s_{1}, t_{1}\right) \equiv$ $J_{2}\left(h^{*}\left(s_{1}, t_{1}\right), t_{1}\right)$. Then, with the help of $J_{2}^{*}$, the original problem (1) reduces to the first-stage problem:

\section{First stage}

Given the constraints (1a) and (1b), we look for an admissible optimal control $a^{*}(\cdot)$ on $\Lambda$ and the associated optimal arrival time $t_{1}^{*} \in \mathscr{T}$ such that

$\max _{\left\{a(\cdot) \in \mathscr{A}, t_{1} \in \mathscr{T}\right\}} V\left(a(\cdot), t_{1}\right) \equiv-J_{1}\left(a(\cdot), t_{1}\right)+J_{2}^{*}\left(s\left(t_{1}\right), t_{1}\right)$,

where the optimal arrival time $t_{1}^{*}$, satisfying (1b), is determined by the acceleration path $a^{*}(\cdot)$, through $\int_{0}^{t_{1}} v(t) \mathrm{d} t=$ $x\left(t_{1}\right)-x(0)=x_{1}$. Since $t_{1}^{*} \in(0, T)$ by assumption, this problem reduces to a standard problem with terminal value (or "salvage" value) $J_{2}^{*}$, free terminal time $t_{1}$ and free end point $s\left(t_{1}\right)$. (For the solution of such a problem, see, for example, Léonard and Long 1992, sec. 7.2 and 7.6)

\section{Second stage: harvesting}

We solve the harvesting problem in this section, and then we solve the problem of the first stage in Section "First stage: optimal travelling-and-harvesting policy". We consider two standard specifications for the growth process of the resource: exponential growth in Section "Exponential growth" and logistic growth in Section "Logistic growth". Exponential growth is a stark abstraction for most real growth processes, of course, as possible density dependence of the growth rate is neglected. The assumption of exponential growth implicitly postulates that possible density dependence of growth becomes effective at population scales beyond the considered abundances. Logistic growth, though, takes this density dependence explicitly into account. Thus, exponential growth may be a reasonable assumption for low abundances, while logistic growth is more descriptive for high abundances. For these reasons, both processes should be considered as complementary. ${ }^{10}$

\section{Exponential growth}

Suppose that the stock of the renewable resource, when left unimpaired, increases at a constant rate: $g(s(t))=s(t)$. Since the stock is reduced by the catch $H(t) \equiv s(t) h(t)$, it evolves according to the differential equation

$\dot{s}(t)=s(t)-h(t) s(t), \quad s\left(t_{1}\right)=s_{1}, \quad \forall t \in \Delta$.

We abstract from discounting for the moment and set $\rho=0$. (We show in Appendix A how our results are affected by the presence of a positive discount rate.) Then, the objective function of Eq. (2b) becomes

$$
\begin{aligned}
& \max _{\{h(\cdot) \in \mathscr{H}\}} J_{2}\left(h(\cdot), t_{1}\right)=\int_{t_{1}}^{T} M h(t) s(t) \mathrm{d} t \\
& \text { s.t. (4a), } s(T) \text { free. }
\end{aligned}
$$

The Hamiltonian of this problem is given by

$$
\mathcal{H}=M h(t) s(t)+\pi(t) s(t)(1-h(t)) .
$$

Since $\mathcal{H}$ is linear in the control $h$, we expect that the optimal solution is of the bang-bang type, with $h$ jumping between the lower and the upper bound, 0 and $\bar{h}$. We now show that such a policy is in fact optimal. To this end, let $\sigma(t) \equiv$ $\partial \mathcal{H} / \partial h=(M-\pi(t)) s(t)$ denote the switching function. Then, the maximum principle yields

$$
\begin{aligned}
h(t) & = \begin{cases}0 & \text { if } \sigma(t)<0, \\
{[0, \bar{h}]} & \text { if } \sigma(t)=0, \\
\bar{h} & \text { if } \sigma(t)>0,\end{cases} \\
\dot{\pi}(t) & =h(t) \pi(t)-M h(t)-\pi(t), \quad \forall t \in \Delta,
\end{aligned}
$$

\footnotetext{
${ }^{10}$ Similar models can be found, for example, in the textbooks of Conrad and Clark (1987), Hocking (1991), and Clark (2010).
} 
along with Eq. (4a) and the transversality condition $\pi(T)=$ $0 .{ }^{11}$ Thus the optimal strategy depends on whether $\pi$ is less or greater than $M$. Next, using $\pi(T)=0$ together with $h(t)=\bar{h}$ for $\pi(t)<M$, Eq. (4b) implies that we cannot end the harvesting period $\Delta$ with $h=0$. Hence, the agent must complete the harvesting period by harvesting at maximal effort, i.e., $h(T)=\bar{h}$.

Moreover, the solution of Eq. (4c) must satisfy

$\pi(t)= \begin{cases}A_{0} e^{-t} & \text { if } h(t)=0 \\ M \frac{\bar{h}}{\bar{h}-1}+A_{1} e^{t(\bar{h}-1)} & \text { if } h(t)=\bar{h} .\end{cases}$

Neither solution achieves the critical value $\pi=M$ more than once. Consequently, there is a unique switching point $\tau,{ }^{12}$ implying that we either have (i) $h(t)=\bar{h}$ for all $t \in \Delta$, or (ii) $h(t)=0$ for all $t_{1} \leq t<\tau$ and $h(t)=\bar{h}$ for all $\tau \leq t \leq T$. Then, along any path with $h=\bar{h}$, the costate variable is given by

$\pi(t)=M \frac{\bar{h}}{\bar{h}-1}\left(1-e^{(1-\bar{h})(T-t)}\right)$

where we used the transversality condition $\pi(T)=0$ to determine the constant $A_{1}=M \bar{h} e^{T(1-\bar{h})} /(1-\bar{h})$. Moreover, the switching time $\tau$ is defined by $\pi(\tau)=M$ so that we obtain from Eq. (5)

$\tau=T-\delta, \quad$ with $\quad \delta \equiv \frac{\log (\bar{h})}{\bar{h}-1}$.

Since $\delta$ is a positive, decreasing and convex function for all values of $\bar{h},{ }^{13}$ we conclude that the larger the maximal harvesting (or effort) rate $\bar{h}$, to which we subsequently also refer to as harvesting capacity, the longer the agent can wait and let the resource grow unimpaired in order to allow for more intense harvesting later. Depending on the sign of $\tau-t_{1}$, or equivalently, on the length of the harvesting period, either of two cases may occur (see Fig. 2):

Case $A: T<\delta+t_{1}$

In this case, the maximal harvesting rate is relatively low, requiring a longer period of harvesting. There is thus no

\footnotetext{
${ }^{11}$ The use of the transversality condition $\pi(T)=0$ requires that $s(T)$ is free. Of course, $s(T)$ is required to be non-negative, so that, in principle, $\pi(T)$ might be positive if $s^{*}(T)=0$. However, it follows from the exponential growth function (4a), that if the resource is "heavily depleted" continuously, in the sense that $h(t)>1$ for all $t>\xi$, for some $\xi>0, s(t)$ converges to zero for $t \rightarrow \infty$. Hence, while, depending on the parameter choice, the residual stock $s(T)$ may be small, the resource never becomes extinct for any finite time $T$, irrespective of the parameter choice.

${ }^{12}$ Alternatively, this observation follows from Eq. (4c), which implies that evaluated at a switching point $\tau$, we have $\dot{\pi}(\tau)=-M$ since $\pi(\tau)=M$ by definition.

${ }^{13}$ For $\bar{h}=1$ we define $\delta=1$ so as to make $\delta$ a continuous function of $\bar{h}$. To see that, in fact, $\lim _{\bar{h} \rightarrow 1} \delta(\bar{h})=1$ apply l'Hôpital's rule.
}

time for "waiting" and choosing a zero harvesting rate for some initial time period. Rather, upon arrival the agent immediately begins harvesting (at the maximal rate), so that there is no policy switch during the harvesting period, but maximal harvesting throughout.

Proposition 1 Let $T<\delta+t_{1}$ and $\bar{h} \neq 1$. Then the optimal harvesting policy is given by

$$
\begin{aligned}
h(t) & =\bar{h}, \quad s(t)=s_{1} e^{(1-\bar{h})\left(t-t_{1}\right)}, \\
\pi(t) & =M \frac{\bar{h}}{\bar{h}-1}\left(1-e^{(1-\bar{h})(T-t)}\right),
\end{aligned}
$$

for all $t \in \Delta$, and the resulting maximised profit amounts to

$J_{2 A}^{*}\left(s_{1}, t_{1}\right) \equiv s_{1} M \frac{\bar{h}}{\bar{h}-1}\left(1-e^{(1-\bar{h})\left(T-t_{1}\right)}\right)$.

Proof The result follows from the preceding analysis.

\section{Case B: $T>\delta+t_{1}$}

In this case, the maximal harvesting rate is relatively high, so that the agent can afford to postpone the start of harvesting. Then, after some idle time-or "waiting" timehas elapsed, harvesting takes place at maximal capacity. During "the waiting period", $\left[t_{1}, \tau\right)$, the stock is left unimpaired and is thus given by $s(t)=s_{1} e^{t-t_{1}}$, so that at the switching time $\tau$ the stock amounts to $s(\tau)=$ $s_{1} e^{\tau-t_{1}}$, which then is the starting value of the stock for the harvesting period $[\tau, T]$. Consequently, for times $t \in[\tau, T]$ the stock equals

$s(t)=A_{2} e^{(1-\bar{h}) t}=s(\tau) e^{(1-\bar{h})(t-\tau)}=s_{1} e^{\bar{h}(\tau-t)+t-t_{1}}$.

Proposition 2 Let $T>\delta+t_{1}$ and $\bar{h} \neq 1$. Then the optimal harvesting policy is given by

$$
\begin{aligned}
& h(t)= \begin{cases}0 & \text { for } t_{1} \leq t<\tau \\
\bar{h} & \text { for } \tau \leq t \leq T\end{cases} \\
& s(t)= \begin{cases}s_{1} e^{t-t_{1}} & \text { for } t_{1} \leq t<\tau \\
s_{1} e^{\bar{h}(\tau-t)+t-t_{1}} & \text { for } \tau \leq t \leq T\end{cases} \\
& \pi(t)= \begin{cases}e^{\tau-t} & \text { for } t_{1} \leq t<\tau \\
M \frac{\bar{h}}{\bar{h}-1}\left(1-e^{(1-\bar{h})(T-t)}\right) & \text { for } \tau \leq t \leq T\end{cases}
\end{aligned}
$$

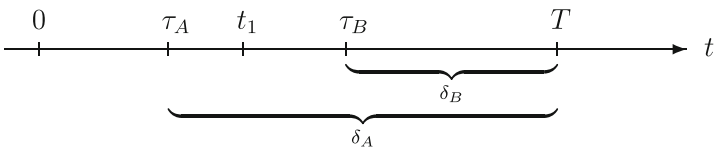

Fig. 2 Case A: early switching time $\tau$, i.e., a small harvesting period $T-t_{1}<\delta$; Case B: late switching time $\tau$, i.e., a large harvesting period $T-t_{1}>\delta$ 

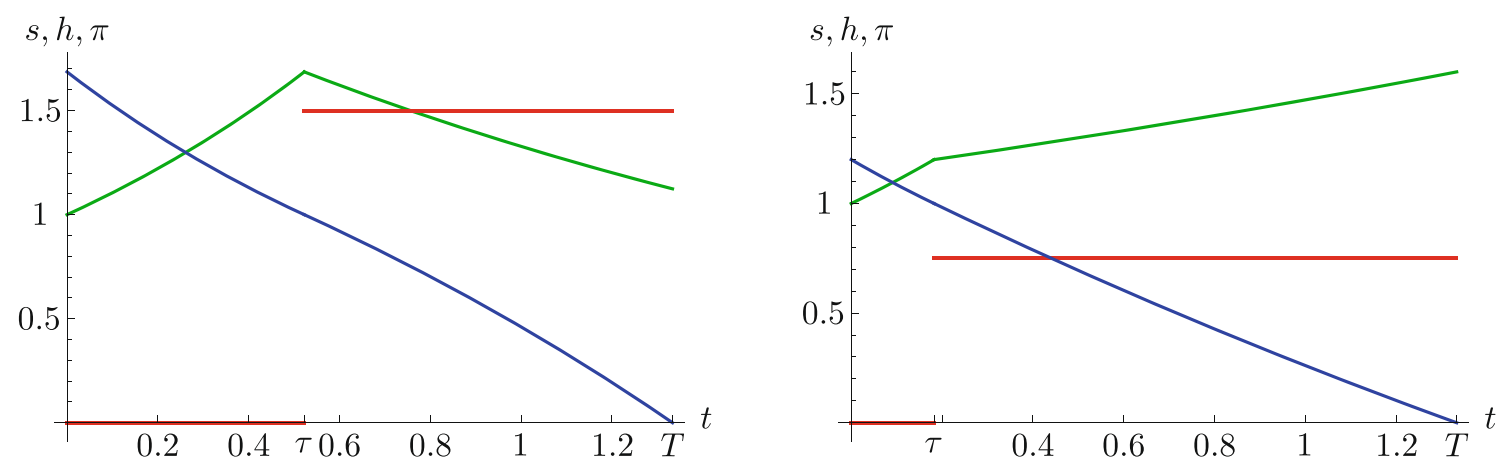

Fig. 3 Optimal harvesting effort in Case B, $T>\delta+t_{1}$ : with $\bar{h}=3 / 2>1$ and thus $\tau=0.5224$ (left diagram), and $\bar{h}=3 / 4<1$ and thus $\tau=0.1826$ (right diagram), both for $t_{1}=0, T=4 / 3$ and $M=1$; with the stock in green, the costate in blue and the control in red

for all $t \in \Delta$, and the maximised profit amounts to

$$
\begin{aligned}
J_{2 B}^{*}\left(s_{1}, t_{1}\right) & \equiv M \frac{\bar{h}}{\bar{h}-1} s_{1} e^{\tau-t_{1}}\left(1-e^{(1-\bar{h})(T-\tau)}\right) \\
& =M s_{1} \bar{h}^{1 /(1-\bar{h})} e^{T-t_{1}} .
\end{aligned}
$$

Proof The result follows from the preceding analysis.

Remark 1 For $T=\delta+t_{1}$, or equivalently, for $\tau=t_{1}$ Case A and Case B coincide.

\section{The case of $\bar{h}=1$}

Finally, the optimal policy for the case $\bar{h}=1$ is obtained by taking the limits of Case A and Case B:

Remark 2 If $\bar{h}=1$, the optimal profit amounts to

$\left.J_{2}^{*}\right|_{\bar{h}=1}\left(s_{1}, t_{1}\right)= \begin{cases}s_{1} M\left(T-t_{1}\right) & \text { if } T \leq \delta+t_{1} \\ s_{1} M e^{T-t_{1}-1} & \text { if } T>\delta+t_{1}\end{cases}$

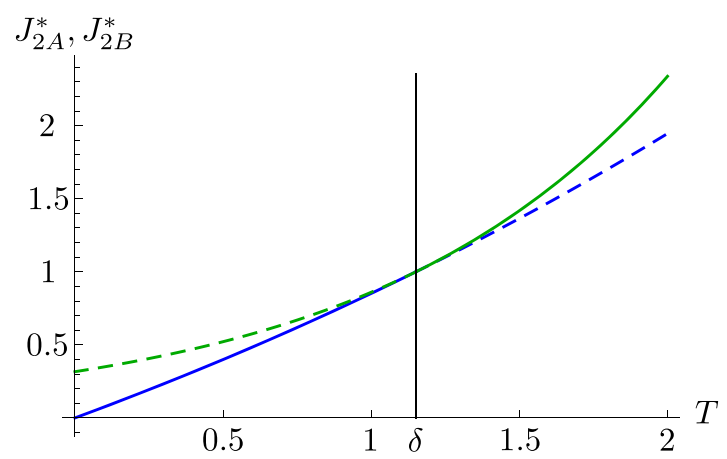

Fig. 4 Maximised profit (solid curves) as a function of the time horizon $T$ : for $\bar{h}=3 / 4<1$, thus $\delta=4 \log (4 / 3)=1.1507$ (left diagram), and for $\bar{h}=3 / 2>1$, and thus $\delta=2 \log (3 / 2)=0.8109$

\section{Discussion}

There is a critical length of the harvesting period given by $\delta=\log (\bar{h}) /(\bar{h}-1)$. If there is plenty of time in the sense that $T-t_{1}>\delta$, there is no harvesting during the initial phase $\left[t_{1}, \tau\right)$ of the harvesting period, but harvesting takes place at the maximum rate $\bar{h}$ during the final phase $[\tau, T]$ of the harvesting period only. In this case, the harvesting technology is so efficient that the agent may wait to let the resource grow further, until harvesting begins. If, however, there is not enough time available, i.e., $T-t_{1} \leq \delta$, the agent is required to harvest at the maximum rate throughout. The relatively low efficiency of the harvesting technology lets the agent harvest as much as possible during the entire harvesting period. Finally, whether the stock increases or decreases during the harvesting process depends on whether the harvesting capacity $\bar{h}$ exceeds or falls short of the growth rate of the stock, which is assumed to be equal to unity here. The situation when $\bar{h}>1$ is depicted in the left diagram of Fig. 3, and when $\bar{h}<1$, in the right diagram (both for $\left.t_{1}=0\right)$.

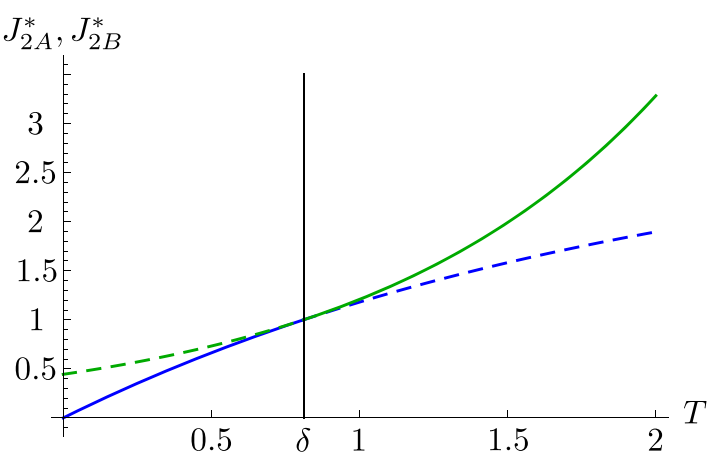

(right diagram): both for $t_{1}=0$ and $M=1$. Since $J_{2 A}^{*}$ (in blue) is defined only for $T \leq \delta$, and $J_{2 B}^{*}$ (in green) is defined only for $T>\delta$, the dashed parts are merely hypothetical. 
Remarkably, the critical length of the harvesting period, $\delta$, depends on the harvesting capacity $\bar{h}$ but is independent of the time horizon; the maximised profit though (in both Case A and B) does depend on $T$. For any given values of the arrival time $t_{1}$ and the stock at arrival $s_{1}$, we find that the profit in Case B is higher than or equal to the profit in Case A, i.e., $J_{2 B}^{*} \geq J_{2 A}^{*}$. This is quite intuitive as a high harvesting capacity is beneficial, allowing for a greater flexibility of the harvesting profile. This is depicted in Fig. 4 for the case $t_{1}=0$. Therein, the vertical line represents the critical time $T=\delta+t_{1}$ for a given value of the harvesting capacity $\bar{h}$, and the red curve depicts the (composed) profit function for varying values of $T$. If time is scarce in the sense that $T-t_{1}<\delta$, Case A applies and the blue curve represents the resulting maximised profit (coinciding with the red curve for values $T<\delta$ ). If there is plenty of time, in the sense that $T>\delta+t_{1}$, Case B applies and the green curve represents the resulting maximised profit (similarly coinciding with the red curve for values $T>\delta+t_{1}$ ).

\section{Logistic growth}

In this section, we modify the growth process of the resource and now explicitly take into account possible density dependence of the growth function. To this end, we assume that the stock obeys a logistic growth process:

$g(s(t))=2 s(t)\left(1-\frac{s(t)}{2}\right), \quad \forall t \in \Delta$,

with a carrying capacity $s^{*}=2 .{ }^{14}$ With this specification, the net growth of the stock is governed by the differential equation

$\dot{s}(t)=g(s(t))-h(t) s(t)=s(t)(2-s(t)-h(t)), \quad \forall t \in \Delta$.

We assume that the resource has been left unimpaired for a sufficiently long time, or even represents a pristine stock (a scenario that is, for example, also considered by Robinson et al. 2008), so that at the arrival time $t_{1}$ the stock equilibrates at its steady state level $s\left(t_{1}\right)=s^{*}=2 .{ }^{15}$

\footnotetext{
${ }^{14}$ This specification is used, for example, in Pindyck (1984), Conrad and Clark (1987), Thieme (2003), Da Lara and Doyen (2008), Polasky et al. (2011) and other applications. We assume a growth rate $r=2$, which may seem high. The numerical results depend on the ratio of the growth rate $r$ and the harvesting capacity $\bar{h}$, with a relatively high (low) growth rate resulting in a higher (lower) final stock. However, since the stock never gets fully depleted by time $T$ (see fn. 11), the particular value of the growth rate does not affect the qualitative results.

${ }^{15}$ Clearly, in finite time the stock cannot fully recover so that the assumption $s\left(t_{1}\right)$ equals the steady state stock $s^{*}=2$ should be understood as an approximation, unless the stock is actually pristine. If we assumed instead that $s\left(t_{1}\right)=s_{0}$ for some value $s_{0} \in(0,2)$, we may have obtained additional cases, namely cases where the agent first refrains from harvesting in order to let the stock grow for some time, and then starts harvesting later.
}

The remaining model is adopted from Section "Exponential growth".

The Hamiltonian of the problem is given by

$\mathcal{H}=M h(t) s(t)+\pi(t) s(t)(2-s(t)-h(t))$.

As in the case of exponential growth, $\mathcal{H}$ is linear in the control $h$. Yet here, as we will see, the optimal solution is not only of the bang-bang type, but may also follow a singular path for some time interval if there is plenty of time (or, equivalently, if $\bar{h}$ is sufficiently large). To this end, let $\sigma(t) \equiv \partial \mathcal{H} / \partial h=(M-\pi(t)) s(t)$ denote the switching function. Then, the maximum principle yields

$$
\begin{aligned}
& h(t)=\left\{\begin{array}{ll}
0 & \text { if } \sigma(t)<0, \\
{[0, \bar{h}]} & \text { if } \sigma(t)=0, \\
\bar{h} & \text { if } \sigma(t)>0,
\end{array} \quad \forall t \in \Delta,\right. \\
& \dot{\pi}(t)=-M h(t)-\pi(t)(2-2 s(t)-h(t)), \quad \forall t \in \Delta,
\end{aligned}
$$

together with Eq. (9a) and the transversality condition $\pi(T)=0$. If we have $\sigma(t)=0$ for some time interval, then the optimal solution contains a singular arc, otherwise we have a bang-bang solution. The next lemma helps to find the specific pattern of the solution.

Lemma $1 \pi\left(t_{1}\right)<M$.

Proof See Appendix B.

It follows from Lemma 1 that the optimal policy rule coincides with the rule obtained for exponential growth of the resource (4b): Since $\pi\left(t_{1}\right)<M$, the optimal path begins with maximal harvesting $h\left(t_{1}\right)=\bar{h}$. Intuitively, since the initial stock equals its maximum level, $s\left(t_{1}\right)=2$, there is no reason to begin with moderate harvesting or even to wait. If time is scarce, relative to the harvesting capacity, the choice of the maximal harvesting effort $h(t)=\bar{h}$ is optimal for all $t \in \Delta$. If, however, there is plenty of time, it is optimal to reduce harvesting in an intermediate time interval, because otherwise harvesting will be completed too early, and the terminal condition that a (marginal) unit of the stock is worthless at the end of the planning horizon, $\pi(T)=0$, will not be met.

Proposition 3 The optimal harvesting policy is given by

$$
\begin{aligned}
& h(t)=\bar{h} \\
& h(t)=\left\{\begin{array}{ll}
\bar{h} & t_{1} \leq t<t_{2}, \\
1 & t_{2} \leq t<t_{3}, \\
\bar{h} & t_{3} \leq t<T,
\end{array} \quad \text { if } \bar{h} \leq \bar{h}_{c}>\bar{h}_{c},\right.
\end{aligned}
$$

with some critical harvesting capacity $\bar{h}_{c}>1$ (depending on $T$ ).

Proof See Appendix B. 
Figure 5 illustrates the two situations characterised in Proposition 3. The left column of the diagrams shows the situation for a low harvesting capacity, while the right column displays the situation for a high harvesting capacity. In each case, the green curve represents the zerocline of $s$, while the blue curve represents the zerocline of $\pi$. The horizontal line at $\pi=M$ is the switching manifold defining the singular path of the optimal control (see Eq. 9b). Since the zerocline of $s$ is vertical at $s=2-\bar{h}$, while the zerocline of $\pi$ reaches the switching manifold at $s=1$, both curves have a point of intersection if, and only if, $\bar{h}<1$ (see left column of diagrams). In this case, a saddle path from $s_{0}=2$ to the unique positive steady state $\left(s_{\infty}, \pi_{\infty}\right)=$ $(2-\bar{h}, \bar{h} /(2-\bar{h}))$ exists. However, this path is not optimal, since the steady state cannot be reached in finite time. Consequently, the optimal path starting from $s\left(t_{1}\right)=2$ lies beneath the saddle path terminating at $\pi(T)=0$ according to the transversality condition. In the lower left diagram the optimal trajectories are drawn for different values of $T$.
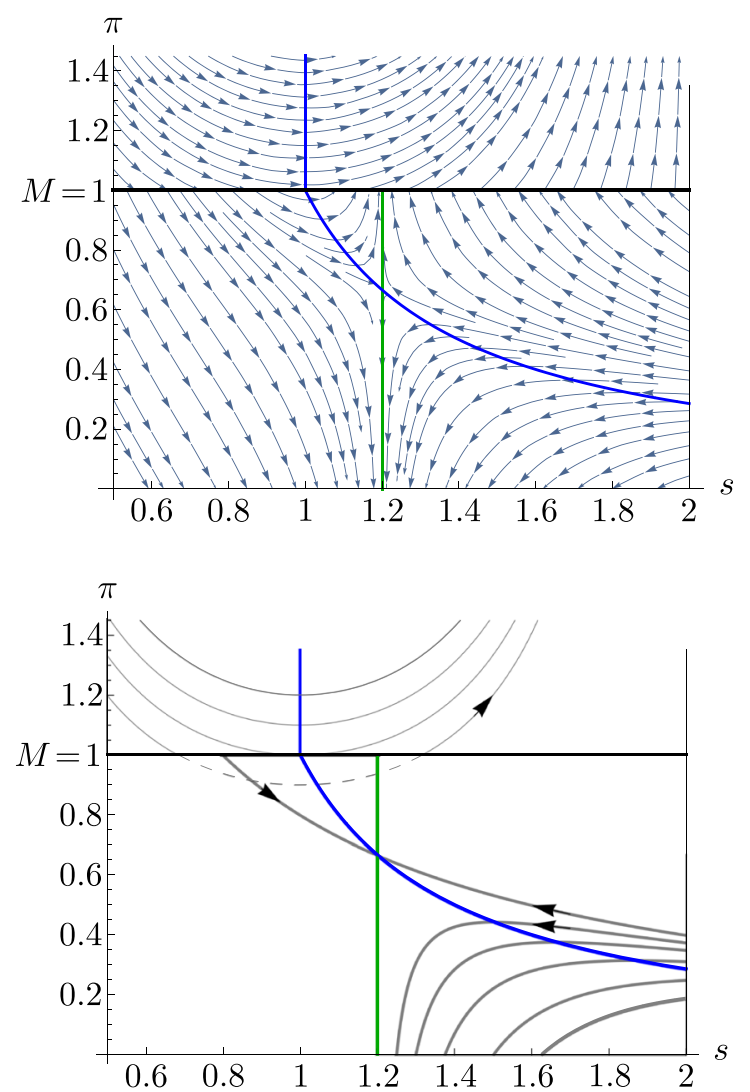

Fig. 5 Phase diagrams (top) and optimal trajectories (bottom) for $\bar{h}=$ 0.8 (left column) and for for $\bar{h}=1.5$ (right column). Bold curves show the optimal trajectories (for different values of $T$ ), the thin curves show the trajectories for $h=0$. The dashed curves represent sub-optimal trajectories, i.e., trajectories that violate Eq. (9b). Blue and green curves
When $\bar{h}>1$, see right column of diagrams in Fig. 5, the zeroclines of $s$ and $\pi$ do not meet, so that in this case a critical trajectory, i.e., a trajectory passing through the point $(1, \mathrm{M})=(1,1)$ exists (see the proof of Proposition 3$)$. The optimal path, starting from $s\left(t_{1}\right)=2$ and terminating at $\pi(T)=0$, lies either beneath the critical trajectory or coincides with it, depending on the harvesting capacity (viz. on the time being available for harvesting). The first case applies if $\bar{h}<\bar{h}_{c}$, the latter if $\bar{h} \geq \bar{h}_{c}$. The next lemma characterises the critical harvesting capacity $\bar{h}_{c}$, and shows that it depends inversely on the time horizon $T$.

Lemma 2 Let $\psi:(1,2] \rightarrow \mathbb{R}_{+}$be defined by

$\bar{h} \mapsto \psi(\bar{h}) \equiv t_{1}+\frac{1}{2-\bar{h}} \log \left(\frac{\bar{h}}{2(\bar{h}-1)^{2}}\right)$

with $\Psi>0, \Psi^{\prime}<0$ and $\Psi^{\prime \prime}>0$. Then, given time $T$, the critical harvesting capacity $\bar{h}_{c}$ is defined as the solution of $T=\psi(\bar{h})$, i.e., $\bar{h}_{c} \equiv \psi^{-1}(T)$. Equivalently, given some
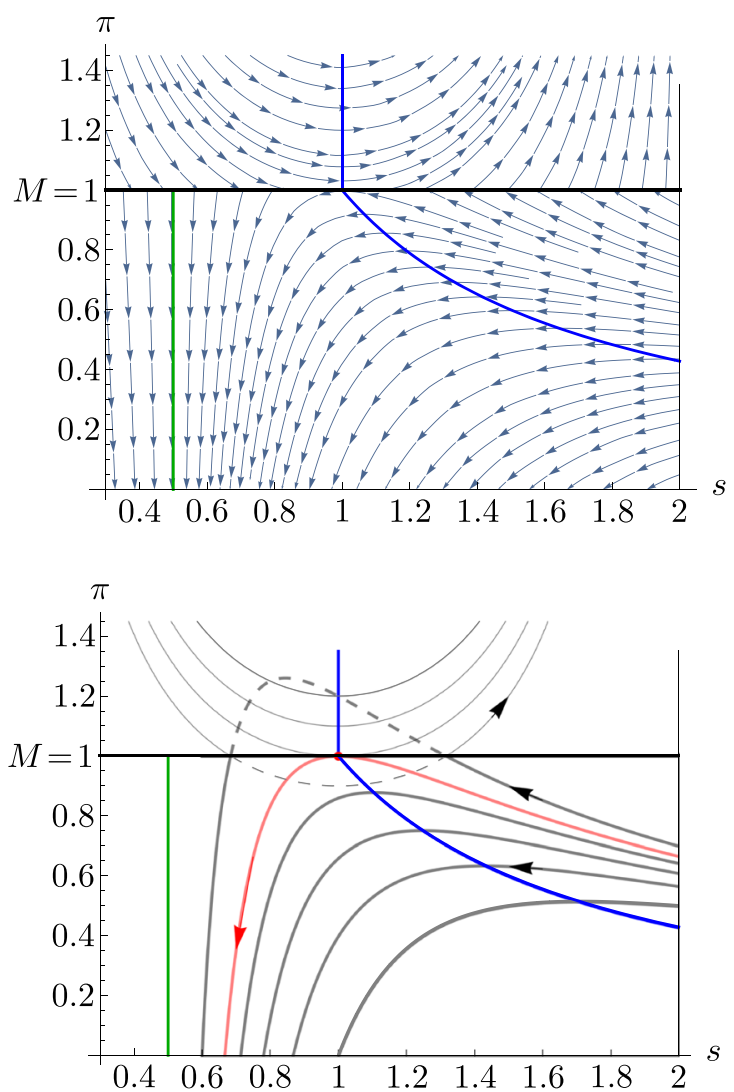

display the zeroclines of $\pi$ and $s$, respectively. The trajectory passing through the point $(1,1)$, displayed in red, represents the critical trajectory; for this trajectory to exist, the harvesting capacity must be sufficiently large, i.e., $h \geq \bar{h}_{c}$ 
harvesting capacity $\bar{h}$, the critical length of the harvesting period is defined by $T_{c} \equiv \psi(\bar{h})$.

Proof See Appendix B.

The intuition for the optimal strategy characterised in Proposition 3 and the critical length of the harvesting period given in Lemma 2 is as follows: In the case $T>T_{c}$, there is too much time for harvesting, implying that if the agent followed the critical path (the red path in the right diagram of Fig. 5), the terminal condition, i.e., the $\pi=0$ line, would be reached too early. Because of this, one might consider following a trajectory lying above the critical one, and thus reaching the $\pi=M$ line at some stock $s>1$. Then however, since the condition $\pi=M$ requires a policy change, one has to dispense with harvesting, and thus to switch to $h=0$. Yet, dispensing with harvesting implies that the stock increases so that the policy is bound to follow an upward-sloping trajectory (a thin path in the right diagram of Fig. 5), implying that both the stock and the costate variable increase; but this makes it impossible to meet the terminal condition $\pi(T)=0$. For that reason, the optimal policy is as follows: Pursue the critical path by harvesting at the capacity limit until the resource is sufficiently reduced and the point $(s, \pi)=(1, M)$ is reached, which happens at time $t_{2}$. Then, upon arrival at $(s, \pi)=(1, M)$, reduce harvesting to the "moderate" effort level $h=1$, which, in view of Eq. (9a) and c, renders both the stock $s$ and its shadow price $\pi$ constant (singular path). This is because unity is the natural growth rate of the resource so that harvesting at exactly this rate represents the sustainable harvesting policy where the stock level is fully preserved. Finally, to complete the optimal path, resume harvesting at the maximal rate so as to arrive at the terminal condition $\pi=0$ at time $T$.

Remark 3 More generally, for an arbitrary growth rate $r$ and an arbitrary carrying capacity $K$, the zerocline of $s$ is vertical at $s=K\left(1-\frac{\bar{h}}{r}\right)$ and the zerocline of $\pi$ is given by $s(t)=\frac{K}{2}\left(1+\frac{h(t)(M-\pi(t))}{r \pi(t)}\right)$. Hence, if $\bar{h} / r$ approaches unity, the zerocline of $s$ becomes vertical at $s=0$. However, the zerocline of $\pi$ meets the switching manifold, i.e., the horizontal $\pi=\mathrm{M}$, at $s=K / 2$, irrespective of the values of $\bar{h}$ and $r$. Since the critical trajectory exists for all parameter values, the minimal stock remaining at time $T$ is determined by the final stock $s(T)$ on the critical trajectory. Consequently, the final stock may become small but does not converge to zero.

\section{Case A: Either $\bar{h}<1$ or $1<\bar{h}<2$ and $T \leq T_{c}$}

In this case, the maximal harvesting effort, relative to the length of the harvesting period—or, equivalently, the length of the harvesting period, relative to the maximal harvesting capacity-is low, $\bar{h}<\bar{h}_{c}=\psi^{-1}(T)$, so that maximal harvesting at rate $\bar{h}$, can be maintained throughout the complete harvesting period. Then, the optimal harvesting strategy is given by:

Proposition 4 Let either $\bar{h}<1$ or $1<\bar{h}<2$ and $T \leq T_{c}$. Then the optimal harvesting policy is given by

$$
\begin{aligned}
& h(t)=\bar{h}, \quad s(t)=\frac{2(\bar{h}-2)}{\bar{h} e^{(\bar{h}-2)\left(t-t_{1}\right)}-2}, \\
& \pi(t)=M \frac{\bar{h}(s(T)-s(t))}{2 s(t)-s(t)^{2}-\bar{h} s(t)},
\end{aligned}
$$

for all $t \in \Delta$, and the resulting maximised profit amounts to $J_{2 A}^{*}\left(t_{1}\right)=\bar{h} M \int_{t_{1}}^{T} s(t) \mathrm{d} t=\bar{h} M \log \left(\frac{2 e^{(2-\bar{h})\left(T-t_{1}\right)}-\bar{h}}{2-\bar{h}}\right)$.

Proof We know from the proof of Proposition 3 that for all sub-critical cases $T<T_{c}$ (or $\bar{h}<\bar{h}_{c}$ ) defined in Eq. (11), we have $h(t)=\bar{h}$ for all $t \in \Delta$. Substituting this, jointly with initial condition $s\left(t_{1}\right)=2$ and the terminal condition $\pi(T)=0$, into Eq. (9a)-(9c) we obtain Eq. (12a).

Remark 4 For the limiting case when $\bar{h} \rightarrow 1$, the resulting profit amounts to $\left.J_{2 A}^{*}\right|_{\bar{h}=1}\left(t_{1}\right)=M \log \left(2 e^{T-t_{1}}-1\right)$.

Remark 5 In the critical case, i.e., when $T=T_{c}$, the optimal profit amounts to

$J_{2 A}^{c}\left(t_{1}\right)=2 \bar{h} M \log \left(\frac{\bar{h}}{\bar{h}-1}\right)$.

\section{Case B: $1<\bar{h}<2$ and $T>T_{c}$}

In this case, the time available for harvesting $T-t_{1}$ is too long such that, given the maximal harvesting capacity, $\bar{h}$, it is not optimal to harvest at the maximal rate all the time, as this implies that the terminal condition $\pi=0$ is met before the final time $T$. Thus, harvesting cannot be maintained at the maximal rate $\bar{h}$ throughout the complete harvesting period, but must be reduced for some time interval.

Proposition 5 Let $1<\bar{h}<2$ and $T>T_{c}$. Then the optimal harvesting policy is given by

$h(t)= \begin{cases}\bar{h} & t_{1} \leq t<t_{2}, \\ 1 & t_{2} \leq t<t_{3}, \\ \bar{h} & t_{3} \leq t<T,\end{cases}$ 

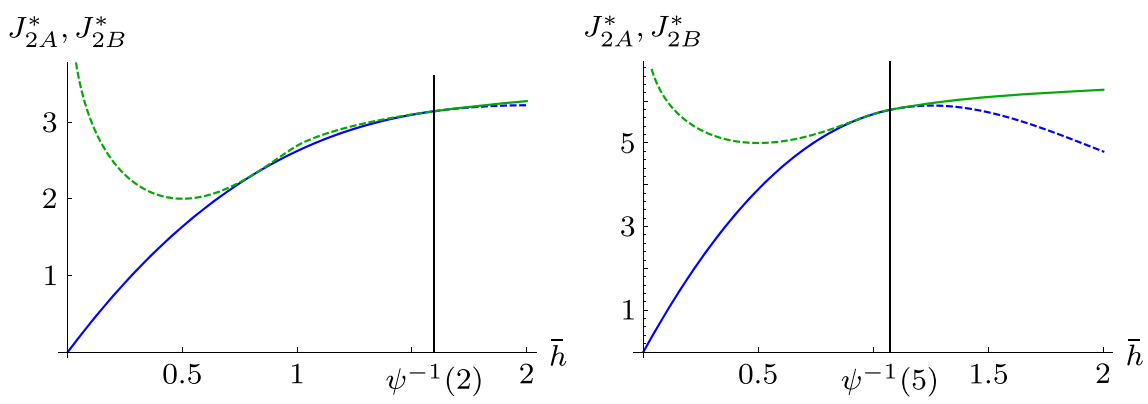

Fig. 6 Maximised profit (solid curves) as a function of the harvesting capacity $\bar{h}$ : with $T=2$, and thus $\bar{h}_{c}=1.59791$ (left diagram); with $T=5$, and thus $\bar{h}_{c}=1.07193$ (middle diagram); and with $T=20$,

with switching times

$t_{2}=t_{1}+\frac{\log \left(\frac{\bar{h}}{2(\bar{h}-1)}\right)}{2-\bar{h}}$ and $t_{3}=T-\frac{\log \left(\frac{1}{\bar{h}-1}\right)}{2-\bar{h}}$.

The resulting profit is given by

$J_{2 B}^{*}\left(t_{1}\right)=$

$M\left[T-t_{1}+2 \bar{h} \log \left(\frac{\bar{h}}{\bar{h}-1}\right)-\frac{1}{2-\bar{h}} \log \left(\frac{\bar{h}}{2(\bar{h}-1)^{2}}\right)\right]$.

Proof See Appendix B.

In the limiting case where $T=T_{c}$, we have $t_{2}=t_{3}$ and the intermediate interval vanishes. More generally, since $\partial t_{2} / \partial \bar{h}<0$ and $\partial t_{3} / \partial \bar{h}>0$, the intermediate interval increases with $\bar{h}$. The reason for this is that a higher harvesting capacity allows the agent to harvest more intensely in the beginning and at the end of the harvesting period, so that harvesting must be reduced in the intermediate time interval in order to avoid too intense harvesting that reduces the stock too quickly. Yet, since we have $h(t)=1$, for all $t \in\left[t_{2}, t_{3}\right)$, the only way to accomplish a lower catch in the intermediate time interval is to extend this interval.

Remark 6 For the limiting case when $\bar{h} \rightarrow 1$, the resulting profit equals $\left.J_{2 B}^{*}\right|_{\bar{h}=1}=M\left[T-t_{1}+\log (2)\right]$; while for the case $\bar{h} \rightarrow 2$, the profit amounts to $\left.J_{2 B}^{*}\right|_{\bar{h}=2}=$ $M\left[T-t_{1}-\frac{3}{2}+\log (16)\right]$. Finally, when the harvesting capacity becomes unbounded, i.e., $\bar{h} \rightarrow \infty$, we obtain $J_{2 B}^{*}=M\left[T-t_{1}+2\right]$. Hence, we have $\left.J_{2 B}^{*}\right|_{\bar{h}=1}$ $<\left.J_{2 B}^{*}\right|_{\bar{h}=2}<\left.J_{2 B}^{*}\right|_{\bar{h}=\infty}$, as expected.

As Remark 6 suggests, the maximised profit function $J_{2}^{*}$ is increasing in the capacity $\bar{h}$; this is depicted in Fig. 6 for $M=1, T=2,5$ and 20 . Therein, the vertical line represents the critical capacity $\bar{h}_{c}=\psi^{-1}(T)$. For values of $\bar{h}<\psi^{-1}(T)$, Case A applies; for values of $\bar{h}>\psi^{-1}(T)$, Case B. The critical values $\bar{h}_{c}=\psi^{-1}(T)$ can be gathered from Eq. (11).

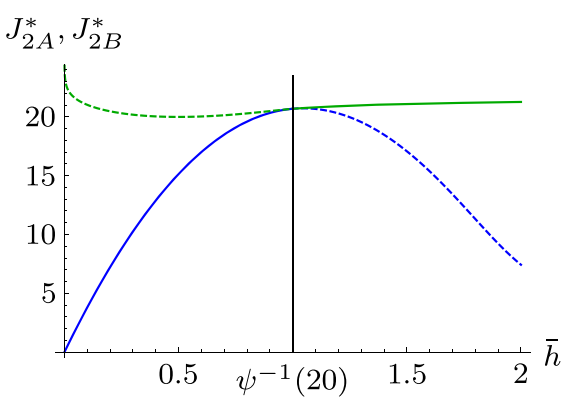

and thus $\bar{h}_{c}=1.00003$ (right diagram); all for $M=1$. Since $J_{2 A}^{*}$ (in blue) is defined only for $\bar{h} \leq \bar{h}_{c}$, and $J_{2 B}^{*}$ (in green) is defined only for $\bar{h}>\bar{h}_{c}$, the dashed parts are merely hypothetical

\section{First stage: optimal travelling-and-harvesting policy}

Having solved the harvesting problem, we now go back in time and solve the travelling problem. We begin our analysis with the simple, hypothetical case of a fixed travelling period in Section "Fixed travelling period", and then continue with acknowledging the subsequent harvesting period and endogenising the arrival time $t_{1}$ in Section "Optimal travelling period". We proceed in this successive manner, as this allows us to spotlight the differences between the solution of the isolated travelling problem (2a) and the solution of the travelling-andharvesting problem (3).

\section{Fixed travelling period}

Assume that the cost of travelling depends linearly on speed $v$ and quadratically on acceleration $a:{ }^{16}$

$K(v, a)=c v+a^{2}$.

Again assuming $\rho=0$, the resulting aggregated travelling cost amounts to

$\int_{0}^{t_{1}}\left(c v(t)+a(t)^{2}\right) \mathrm{d} t$.

(In Appendix A we explore the effects of a positive discount rate.) Acknowledging the constraints $\dot{x}(t)=v(t), \dot{v}(t)=$ $a(t)$, and $\dot{s}(t)=g(s(t))$, we obtain the Hamiltonian

$\mathcal{H}_{1}=-c v(t)-a(t)^{2}+\pi_{2}(t) a(t)+\pi_{1}(t) v(t)$.

\footnotetext{
${ }^{16} \mathrm{~A}$ linear-quadratic dependence of fuel consumption on the kinematic variables velocity and acceleration (along with the characteristics of the road, motor data etc.) is typically used and has been empirically tested in transport economics. See, for example, Ahn et al. (2002), Bifulco et al. (2015), and Wörz and Bernhardt (2017) and the references therein.
} 
For ease of tractability, we assume that there are no bounds on the control $a$-yet, we will drop this assumption in Appendix A. The familiar maximum principle then yields

$$
\begin{aligned}
x(t) & =\frac{t^{2}}{12}\left(3 K_{1}-K_{2} t+c t\right), \\
\pi_{1}(t) & =K_{2}, \\
v(t) & =\frac{t}{4}\left(2 K_{1}-K_{2} t+c t\right), \\
\pi_{2}(t) & =K_{1}+t\left(c-K_{2}\right),
\end{aligned}
$$

with $K_{1}$ and $K_{2}$ constants. Together with the boundary conditions $x(0)=v(0)=v\left(t_{1}\right)=0$ and $x\left(t_{1}\right)=x_{1}$, we obtain:

Proposition 6 Given arrival time $t_{1}$, the optimal travelling policy is given by

$$
\begin{aligned}
x(t) & =\frac{t^{2}\left(3 t_{1}-2 t\right) x_{1}}{t_{1}^{3}}, \quad v(t)=\frac{6 t\left(t_{1}-t\right) x_{1}}{t_{1}^{3}}, \quad a(t)=\frac{6\left(t_{1}-2 t\right) x_{1}}{t_{1}^{3}}, \\
\pi_{1}(t) & =c+\frac{24 x_{1}}{t_{1}^{3}}, \quad \pi_{2}(t)=\frac{12\left(t_{1}-2 t\right) x_{1}}{t_{1}^{3}},
\end{aligned}
$$

and the minimised objective function equals

$J_{1}^{*}\left(t_{1}\right)=\int_{0}^{t_{1}}\left(a(t)^{2}+c v(t)\right) \mathrm{d} t=c x_{1}+\frac{12 x_{1}^{2}}{t_{1}^{3}}$.

Proof The result follows from the preceding analysis.

Since $J_{1}^{*}$ enters the objective function negatively, the value of the maximised Hamiltonian equals

$\mathcal{H}_{1}^{*}\left(t_{1}\right) \equiv \mathcal{H}_{1}\left(s\left(t_{1}\right), a\left(t_{1}\right), \pi\left(t_{1}\right), t_{1}\right)=-\frac{\mathrm{d} J_{1}^{*}\left(t_{1}\right)}{\mathrm{d} t_{1}}=\frac{36 x_{1}^{2}}{t_{1}^{4}}$

The acceleration of the vehicle and its resulting speed are depicted in Fig. 7 for varying arrival times $t_{1}$.

\section{Optimal travelling period}

In Section "Fixed travelling period" we assumed that the arrival time $t_{1}$ is fixed. However, the agent may choose

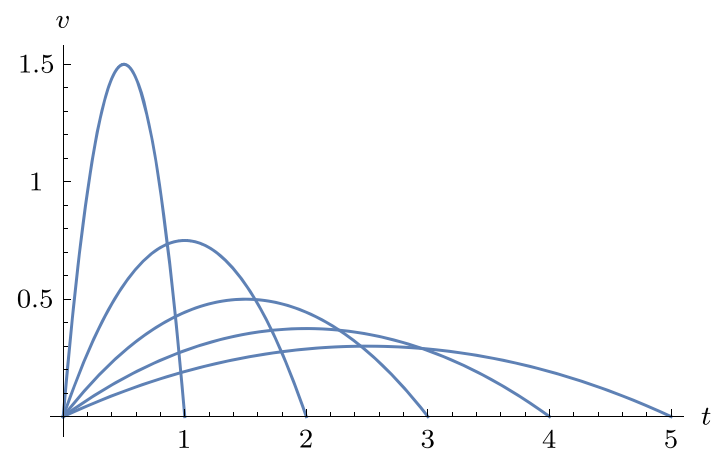

the length of the travelling period and, concordantly, the beginning of the harvesting period. In order to determine the optimal policy for the travelling-and-harvesting problem, two different effects must be taken into account, and the associated conditions have to be added to those of the pure travelling decision. First, the growth process of the resource during the travelling period must be acknowledged, and the corresponding necessary optimality condition needs to be added to the canonical system:

$$
\begin{gathered}
\dot{s}(t)=g(s(t))= \begin{cases}2 s(t)-s^{2}(t) & \text { logistic } \\
s(t) & \text { exponential, }\end{cases} \\
\dot{\pi}(t)=-\frac{\partial \mathcal{H}_{1}}{\partial s(t)}= \begin{cases}-2 \pi(t)(1-s(t)) & \text { logistic } \\
-\pi(t) & \text { exponential. }\end{cases}
\end{gathered}
$$

Next, the terminal time $t_{1}$ and the endpoint, i.e., the stock at the terminal time, $s\left(t_{1}\right)$ of the travelling problem are free and may be chosen in an optimal way. While the arrival time $t_{1}$ determines the length of the harvesting period $\Delta$, the endpoint $s\left(t_{1}\right)=s_{1}$ determines the initial value of the growth process for the harvesting problem. Together, both effects determine the maximal value $J_{2}^{*}\left(s_{1}, t_{1}\right)$ of the harvesting period, which in turn represents the terminal value (or 'salvage' value) of the compound problem (3). However, the arrival time $t_{1}$ coincidentally also determines the endpoint $s_{1}=s\left(t_{1}\right)$, and for this reason we do not have two, but only one transversality condition representing both effects: the direct effect of the arrival time on the length of the harvesting period $\Delta$, and the effect of $t_{1}$ on the stock at the beginning of that period $s\left(t_{1}\right)$.

To derive a necessary condition for the optimal choice of the arrival time $t_{1}$, we first have to substitute the transversality condition $s_{1}=s\left(t_{1}\right)=s_{0} e^{t_{1}}$, into $J_{2}^{*}$, to obtain, with slight abuse of notation, $J_{2}^{*}\left(t_{1}\right) \equiv J_{2}^{*}\left(s\left(t_{1}\right), t_{1}\right)$. Then, defining $V\left(t_{1}\right) \equiv-J_{1}^{*}\left(t_{1}\right)+J_{2 A}^{*}\left(t_{1}\right)$ and using Eq. (18), the associated necessary condition for the free terminal time of the travelling problem $t_{1}$ reads as

$$
\frac{\mathrm{d} V\left(t_{1}^{*}\right)}{\mathrm{d} t_{1}} \equiv-\frac{\mathrm{d} J_{1}^{*}\left(t_{1}^{*}\right)}{\mathrm{d} t_{1}}+\frac{\mathrm{d} J_{2}^{*}\left(t_{1}^{*}\right)}{\mathrm{d} t_{1}} \equiv \mathcal{H}_{1}^{*}\left(t_{1}^{*}\right)+\frac{\mathrm{d} J_{2}^{*}\left(t_{1}^{*}\right)}{\mathrm{d} t_{1}}=0 .
$$

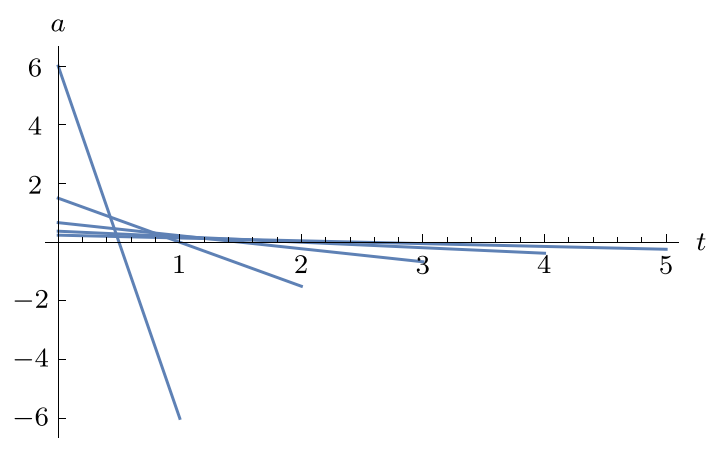

Fig. 7 Speed $(v)$ and acceleration $(a)$ for $t_{1}=1, \ldots, 5\left(c=1 / 10, x_{1}=1\right)$ 
(Condition (20) represents a modification of the usual necessary condition for the free terminal time, as provided, for example, by Léonard and Long 1992, Theorem 7.6.1.) With the help of Eq. (20) we are now able to calculate the optimal travelling-and-harvesting policy. We do this for both growth functions specified above.

\section{Optimal travelling-and-harvesting policy for exponential growth}

Acknowledging the transversality conditions, the following conditions have to be added to the canonical system:

$s(t)=s_{0} e^{t}, \quad \pi(t)=M\left(\frac{1}{\bar{h}}\right)^{\frac{1}{\bar{h}-1}} e^{T-t}$.

We next show that $t_{1}$ must not be smaller than the switching time $\tau$, so that harvesting begins immediately upon arrival. Intuitively, this is because a premature arrival is costly without yielding any additional profit, as we initially have $h(t)=0$ in Case B. Thus, a policy that implies Case B is never optimal, so that Case A must apply for the optimal policy; correspondingly, the maximised value function of the harvesting problem is given by Eq. (7b).

Proposition 7 In the optimal travelling-and-harvesting policy, the arrival takes place after the switching time $\tau$, i.e., $t_{1}^{*}>\tau$, and thus Case A applies. Then, the optimal harvesting policy is characterised by Proposition 1, while the optimal travelling policy is given by Proposition 6. Hence, the resulting profit from the optimal travelling-andharvesting policy is given by

$$
\begin{aligned}
& V\left(t_{1}^{*}\right) \equiv J_{2 A}^{*}\left(t_{1}^{*}\right)-J_{1}^{*}\left(t_{1}^{*}\right)= \\
& s_{0} M e^{t_{1}^{*}} \frac{\bar{h}}{\bar{h}-1}\left(1-e^{(\bar{h}-1)\left(t_{1}^{*}-T\right)}\right)-\left(\frac{12 x_{1}^{2}}{\left(t_{1}^{*}\right)^{3}}+c x_{1}\right),
\end{aligned}
$$

where the optimal arrival time $t_{1}^{*}$ is a function of $\bar{h}$ and $T$, implicitly defined by

$$
\frac{\mathrm{d} V\left(t_{1}^{*}\right)}{\mathrm{d} t_{1}}=\frac{36 x_{1}^{2}}{\left(t_{1}^{*}\right)^{4}}-s_{0} M e^{t_{1}^{*}} \frac{\bar{h}}{\bar{h}-1}\left(\bar{h} e^{(\bar{h}-1)\left(t_{1}^{*}-T\right)}-1\right)=0 .
$$

\section{Proof See Appendix B.}

Lemma 3 The derivative of the maximised value function of the harvesting problem is determined by the switching point $\tau$ :

$\frac{\mathrm{d} J_{2 A}^{*}\left(t_{1}\right)}{\mathrm{d} t_{1}} \lesseqgtr 0 \quad \Leftrightarrow \quad T-t_{1} \gtreqless \delta \equiv \frac{\log (\bar{h})}{\bar{h}-1} \quad \Leftrightarrow \quad \tau \gtreqless t_{1}$.

Proof See Appendix B.
Since $\delta$ is a decreasing function of $\bar{h}$, the derivative of $J_{2 A}^{*}$ is positive for large, and negative for small values of $\bar{h}$. Now, the condition $\mathrm{d} J_{2 A}^{*} / \mathrm{d} t_{1}=0$ determines the optimal arrival time in the absence of any travelling cost. If the harvesting capacity, when compared with the length of the harvesting period $\Delta \equiv T-t_{1}$, is large, a given volume of harvest can be collected in a shorter time interval, thus giving scope for a later arrival. Since a later arrival, leaves the resource with more time to grow, a higher harvesting capacity allows the agent to postpone the arrival time. Conversely, when the harvesting capacity is relatively low, postponing the start of the harvesting activity is unattractive, as the agent will be unable to benefit from the higher stock due to the limited harvesting capacity. The optimal arrival time balances the benefits from an earlier and a later arrival.

Corollary 1 In the absence of any travelling cost, the optimal arrival time equals the switching time, i.e., $t_{1}^{\circ}=$ $\tau \equiv T-\delta$.

We thus find that the optimal arrival time $t_{1}^{*}$ is chosen so that the harvesting activity begins immediately upon arrival (Case A). In other words, the optimal arrival time is relatively late in the sense $T-t_{1}^{*}<\delta$ or $t_{1}^{*}>\tau$, so that the agent begins with harvesting at the maximum rate immediately at time $t_{1}$. This is because an early arrival results in higher travelling costs and curtails the time for further growth. Both effects are unwelcome, so early arrival should be avoided. The determination of the optimal arrival time $t_{1}^{*}$ and the switching time $\tau$ is illustrated in Fig. 8 .

It follows from Proposition 7 and Corollary 1 that higher travelling costs imply later arrival, and that the optimal arrival time $t_{1}^{*}$ exceeds the optimal arrival time when travelling is costless $t_{1}^{\circ}$, which in turn coincides with the

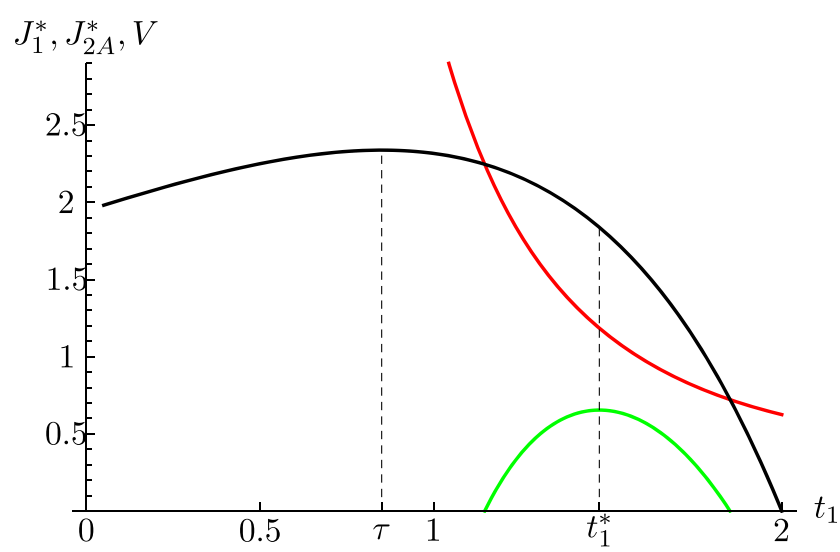

Fig. 8 Exponential growth: revenue function $J_{2 A}^{*}$ (black), cost function $J_{1}^{*}$ (red), and profit function $V$ (green), for $M=1, s_{0}=1$, $T=2, c=1 / 2, x_{1}=1 / 2$ and $\bar{h}=3 / 4$, yielding switching time $\tau=0.84927$, the optimal arrival time $t_{1}^{*}=1.47525$ and the net profit $V\left(t_{1}^{*}\right)=0.65429$ 
switching time: $t_{1}^{*}>t_{1}^{\circ}=\tau \equiv T-\delta$. Conversely, the optimal length of the harvesting period $\Delta^{*} \equiv T-t_{1}^{*}$ (assuming $T>\Delta^{*}$ ) is smaller than the harvesting period the agent would have chosen in the absence of any travelling $\operatorname{cost} \Delta^{\circ} \equiv T-t_{1}^{\circ}$.

\section{Optimal travelling-and-harvesting policy for logistic growth}

In the case of logistic growth we assumed that the resource was left unimpaired for a sufficiently long time, so that upon arrival the stock rests at its steady state level $s\left(t_{1}\right)=$ $s_{1}=s^{*}$. Consequently, in this case the arrival time $t_{1}$ can be chosen without affecting $s_{1}$, but still we have to consider two cases: Case A and Case B.

\section{Case A: either $\bar{h}<1$ or $1<\bar{h}<2$ and $T \leq T_{c}$}

In this case, the optimal harvesting policy is characterised by Proposition 4; and the optimal travelling policy, by Proposition 6. Hence, the functions $J_{2 A}^{*}\left(t_{1}\right)$ and $J_{1}^{*}\left(t_{1}\right)$ are given by Eqs. (12b) and (17), respectively. Moreover, the derivative of $J_{2 A}^{*}\left(t_{1}\right)$ equals

$\frac{\mathrm{d} J_{2 A}^{*}\left(t_{1}\right)}{\mathrm{d} t_{1}}=-\frac{2(\bar{h}-2) \bar{h} M}{\bar{h} e^{(\bar{h}-2)\left(T-t_{1}\right)}-2} \leq 0$,

which is negative for all $0<\bar{h} \leq 2$, as the numerator and the denominator are both negative, and $\lim _{\bar{h} \nearrow_{2}} \mathrm{~d} J_{2 A}^{*}\left(t_{1}\right) / \mathrm{d} t_{1}=$ $-4 M /\left(2\left(T-t_{1}\right)+1\right)<0$; moreover, $\mathrm{d} J_{2 A}^{*}\left(t_{1}\right) / \mathrm{d} t_{1}=$ 0 for $\bar{h}=0$. Since $\mathrm{d} J_{1}^{*}\left(t_{1}\right) / \mathrm{d} t_{1}$ is also negative, the optimal arrival time is determined by Eq. (20), and the optimal travelling-and-harvesting policy is characterised as follows:

Proposition 8 Let either $\bar{h}<1$ or $1<\bar{h}<2$ and $T \leq T_{c}$. Then, the optimal harvesting policy is $h(t)=\bar{h}$ for all

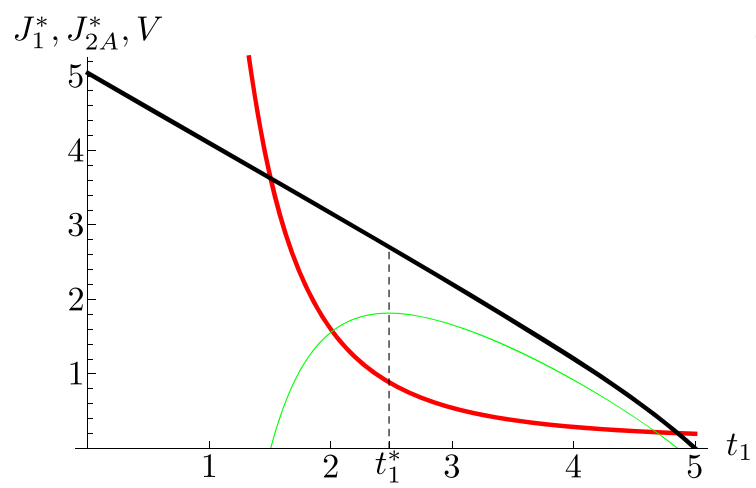

Fig. 9 Logistic growth: value function $J_{2}^{*}$ (black), cost function $J_{1}^{*}$ (red), and profit function $V$ (green), for $M=1, T=5$ and $c=1 / 10$. Case A (left): $\bar{h}=3 / 4$, optimal arrival time $t_{1}^{*}=2.4793$. Case B $t \in \Delta$, and the resulting profit from the optimal travellingand-harvesting policy, $V\left(t_{1}^{*}\right) \equiv J_{2 A}^{*}\left(t_{1}^{*}\right)-J_{1}^{*}\left(t_{1}^{*}\right)$, is given by

$V\left(t_{1}^{*}\right)=\bar{h} M \log \left(\frac{2 e^{(2-\bar{h})\left(T-t_{1}^{*}\right)}-\bar{h}}{2-\bar{h}}\right)-\left(c x_{1}+\frac{12 x_{1}^{2}}{\left(t_{1}^{*}\right)^{3}}\right)$,

where $t_{1}^{*}$ is a function of $\bar{h}$ and $T$, implicitly defined by

$$
\frac{\mathrm{d} V\left(t_{1}^{*}\right)}{\mathrm{d} t_{1}}=\frac{36}{\left(t_{1}^{*}\right)^{4}}-\frac{2(\bar{h}-2) \bar{h} M}{\bar{h} e^{(\bar{h}-2)\left(T-t_{1}^{*}\right)}-2}=0 .
$$

Proof The result follows from the preceding analysis.

The functions $J_{1}^{*}\left(t_{1}\right)$ and $J_{2 A}^{*}\left(t_{1}\right)$ are depicted in Fig. 9 for a low (left diagram) and a high (right diagram) harvesting capacity, with $T=5$ and $M=1$. Setting $\bar{h}=$ $3 / 4$, the optimal arrival time equals $t_{1}^{*}=2.4793$ yielding a net profit equal to $V\left(t_{1}^{*}\right) \equiv J_{2 A}^{*}\left(t_{1}^{*}\right)-J_{1}^{*}\left(t_{1}^{*}\right)=1.8161$. Observe that Case A actually materialises for $T=5$ with $M=1$ and $c=1 / 10$. (Compare the middle diagram in Fig. 6.)

\section{Case B: $1<\bar{h}<2$ and $T>T_{c}$}

In this case, the harvesting capacity $\bar{h}$ exceeds the critical value $\bar{h}_{c}$. Then, the optimal harvesting policy is characterised by Proposition 5, and the associated profit is given by Eq. (14b). Accordingly, the derivative of the value function equals $\mathrm{d} J_{2 B}^{*}\left(t_{1}\right) / \mathrm{d} t_{1}=-M$, and therefore the optimal arrival time $t_{1}^{*}$ can be calculated explicitly:

Proposition 9 Let $1<\bar{h}<2$ and $T>T_{c}$. Then, the optimal harvesting policy is characterised by Proposition 5 ,

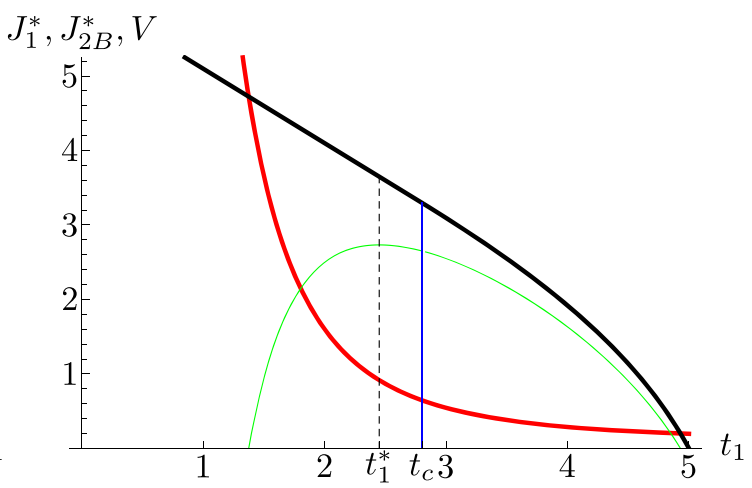

(right): $\bar{h}=3 / 2$, optimal arrival time $t_{1}^{*}=\sqrt{6}=2.4495$ with critical arrival time $t_{c}=5-2 \log (3)=2.8028$ (blue) 

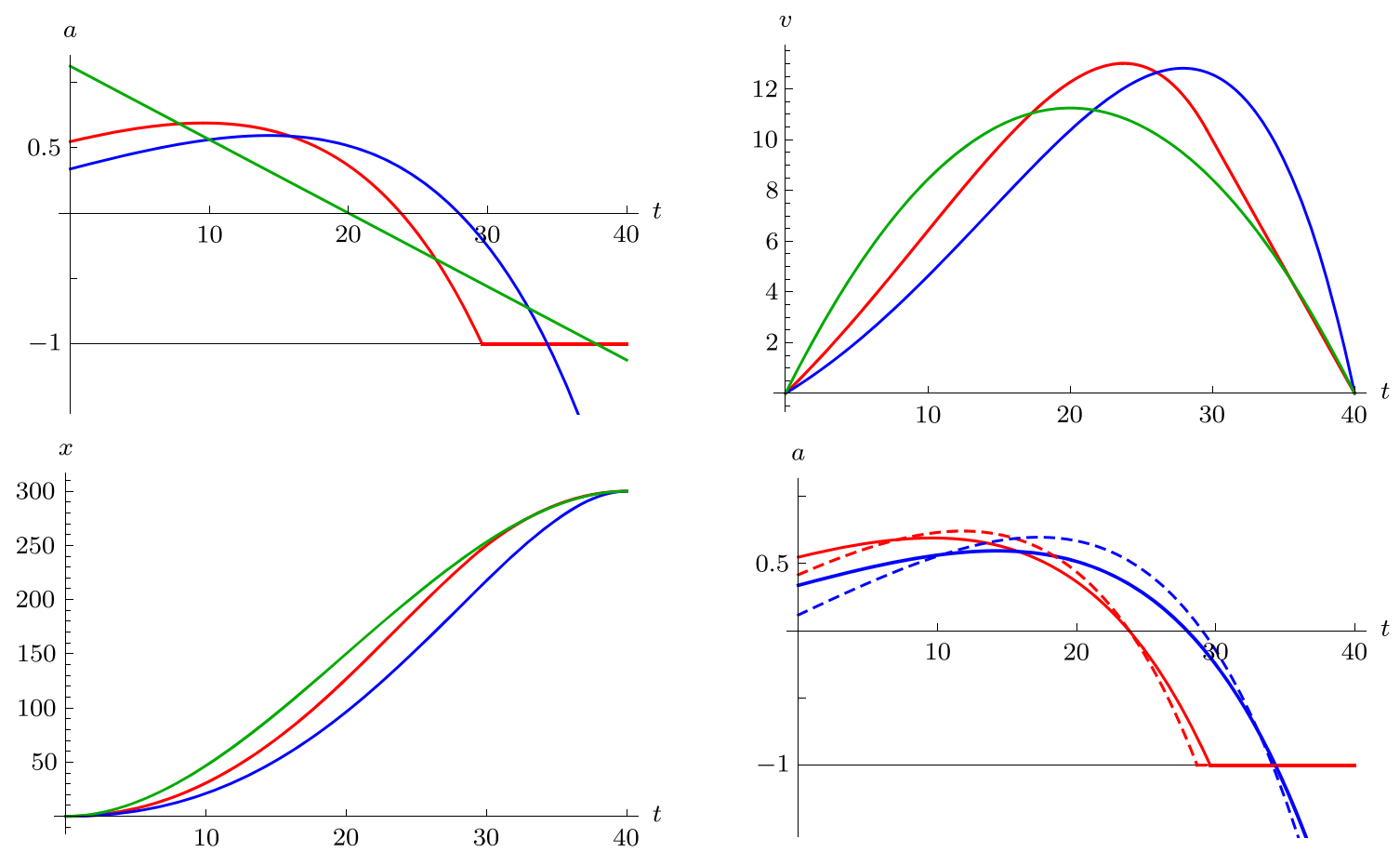

Fig. 10 Optimal acceleration (upper left), speed (upper right) and position (lower left), with (red) and without (blue) bounds on acceleration for $\rho=1 / 20$; and, for comparison, without bounds for $\rho=0$ (green).

Lower right: optimal acceleration for $c=1 / 10$ (bold curves) and $c=1 / 5$ (dashed curves) for $\rho=1 / 20$

and the resulting profit from the optimal travelling-andharvesting policy, $V\left(t_{1}^{*}\right) \equiv J_{2 B}\left(t_{1}^{*}\right)-J_{1}\left(t_{1}^{*}\right)$, is given by

$$
\begin{aligned}
& V\left(t_{1}^{*}\right)= \\
& M\left[T-t_{1}^{*}+2 \bar{h} \log \left(\frac{\bar{h}}{\bar{h}-1}\right)-\frac{1}{2-\bar{h}} \log \left(\frac{\bar{h}}{2(\bar{h}-1)^{2}}\right)\right] \\
& -\left(c x_{1}+\frac{12 x_{1}^{2}}{\left(t_{1}^{*}\right)^{3}}\right)
\end{aligned}
$$

with $t_{1}^{*}=\sqrt{6 x_{1} / \sqrt{M}}$.

Proof The result follows from the preceding analysis.

This scenario is depicted for $\bar{h}=3 / 2$ (and $T=5$, $c=1 / 10, M=1)$ in the right diagram of Fig. 9, where the value function $J_{2 B}^{*}\left(t_{1}\right)$ has slope -1 for all arrival times $t_{1}<t_{c}$. (Recall that for these parameter values, Case B results, which is illustrated in the second diagram in Fig. 6.) With these parameters, the optimal solution is given by $t_{1}^{*}=$ 2.4495 yielding a net profit of $J_{2 B}\left(t_{1}^{*}\right)-J_{1}\left(t_{1}^{*}\right)=2.7326 .{ }^{17}$

Finally, the optimal arrival time for the case that travelling is costless is the same for both Case A and Case B:

\footnotetext{
${ }^{17}$ With a later starting time $t_{1}>t_{c} \equiv T-T_{c}=5-2 \log (3)=2.8028$, Case A would become relevant, as the time left is less than the minimal time interval required for harvesting in Case $\mathrm{B}, T_{c}=2 \log (3)=$ 2.1972 .
}

Corollary 2 In the absence of travelling costs, the optimal arrival time is equal to the earliest (physically) feasible arrival time, i.e., $t_{1}^{\circ}=t_{\min }$.

Proof The result follows from the fact that in both Case A and Case B, the derivative of $J_{2}$ is negative for any $h>0$, and hence the optimal arrival time $t_{1}^{\circ}$ is chosen minimally.

\section{Robustness of the results}

In the previous section, we abstracted from discounting and possible constraints on acceleration. We therefore now discuss the robustness of our results with respect to theses assumptions, and briefly sketch the modifications implied by a positive discount rate and bounds on acceleration.

As shown in Appendix A, we may generalise our optimal travelling result: Proposition 10, generalising Proposition 6 , shows that for a fixed arrival time $t_{1}$, the optimal acceleration depends linearly on the travelling cost parameter $c$, and consequently the minimised travelling cost $J_{1}^{*}$ is a polynomial of $c$ of order 2. Similarly, while in the absence of discounting acceleration depends linearly on time, acceleration becomes a non-linear function of time once the discount rate is positive. Intuitively, a positive discount rate requires a more sophisticated travelling policy: Discounting lets the agent postpone part of the travelling 
cost by shifting the acceleration profile towards the future. In this way, the location of the resource will be reached at the same time, yet at lower cost.

This result can even be generalised to the case of a binding lower bound on acceleration. With positive discounting, the agent again seeks to postpone acceleration to the future. However, a lower bound inhibits this costshifting effect as the agent is required to slow down earlier, and thus must accelerate earlier. Hence, the cost-shifting effect induced by discounting is mitigated by bounds on acceleration. Corollary 3 (see Appendix A) formally characterises this cost-shifting effect.

We illustrate the effects of a positive discount rate and of a lower bound on acceleration for the travelling profile by means of an example, which is used to draw Fig. 10.

Example In order to illustrate our results, we apply the parameter specification: $t_{1}=40, x_{1}=300, \rho=1 / 20, c=$ $1 / 10$. For this specification, the unbounded solution hits the lower bound at time $t=34.2818$ (see blue case in Fig. 10); while the resulting optimal hitting time equals $\xi=29.5984$. Combining the optimal control before and after the hitting time, we obtain

$a(t)= \begin{cases}\frac{1}{2}\left(e^{t / 20}(3.095-0.1046 t)-2\right) & 0 \leq t \leq \xi=29.599, \\ -1 & \xi<t \leq t_{1}=40,\end{cases}$

with minimal travelling cost amounting to $\bar{J}_{1}^{*}=18.4648$. The optimal solution is illustrated by the red trajectories in Fig. 10. As expected, compared with $J_{1}^{*}=16.7095$ for the case of an unbounded control, the presence of the bound on acceleration results in an increase in the minimal travelling cost.

We may also compare our result for a positive discount rate with the case $\rho=0$, displayed by the green trajectories in Fig. 10. In the absence of discounting, we obtain $\left.J_{1}^{*}\right|_{\rho=0}=375 / 8=46.875$, so that discounting makes part of the cost vaporise. This can also be seen in Fig. 10 by comparing the blue with the green paths: a positive discount rate makes the agent initially move more slowly and speed up later so that part of the travelling cost is shifted to the future. (In case of bounds on the control, such cost shifting becomes limited so that some part of the travelling cost must be incurred earlier, compare the red paths.) This costshifting effect is the more pronounced the higher the cost of speed is: compare the optimal acceleration path for $c=$ $1 / 10$ (bold curves) with that for $c=1 / 5$ (dashed curves) in the lower right diagram of Fig. 10.

\section{Conclusion}

In this paper we contribute to the theory of spatial resource economics by explicitly taking into account the fact that in many real-world situations, the agent has to travel to the location of the resource before being able to harvest. Recent papers acknowledge the requirement of an agent to travel to the resource: see Robinson et al. (2008), Belyakov et al. (2015), and Sirén and Parvinen (2015) and others. However, in those models the travelling and the harvesting activity are either inversely related by assumption (so called search models), or harvesting is done in an en passant manner where the harvesting yield is collected while travelling. In both types of models the travelling and the harvesting decisions are linked so that effectively there is only one decision to be made. Only disentangling these two activities allows the agent to make two discrete decisions.

Behringer and Upmann (2014) and Zelikin et al. (2017) disentangled both activities while maintaining the en passant harvesting framework. We follow a different route here: In our two-stage travelling-and-harvesting model, the agent has to travel to the location of the resource in order to harvest. With travelling preceding harvesting and both activities being time-consuming, they are not only disentangled but are also mutually exclusive activities, i.e., separated in time. Still, the travelling problem and the subsequent harvesting problem are closely linked: The arrival time at the location of the resource becomes the crucial decision for the optimal harvesting policy as the speed of travelling determines both the required travelling profile and the start of the harvesting period. Accordingly, there is the need for the agent to weigh the benefits and costs of an early arrival: The benefit of a short travel and thus an early arrival is that it leaves more time for harvesting, and may thus offer the opportunity for a larger aggregate yield from harvesting. This, however, comes at the disadvantage of higher pecuniary travelling cost, and a shorter time for the resource to grow until arrival, and thus less beneficial initial conditions for harvesting. The optimal travelling-andharvesting decision has to balance those benefits and costs.

We are able to analytically characterise the control programme for this combined travelling-and-harvesting problem, employing recent tools from two-stage dynamic optimization theory. To this end, we analyse the two subproblems in reverse order: we first solve the harvesting problem (Proposition 1-5), and then the preceding travelling problem (Proposition 6) taking into account the optimal harvesting policy of the second stage. This allows us to highlight the critical linkage between both stages and thus to characterise the resulting optimal yield from the resource. We derive the optimal policy for two different growth processes of the resource stock: exponential growth (Proposition 7) and logistic growth (Propositions 8 and 9).

The crucial parameters that determine the optimal policy are the harvesting capacity and the length of the planning period, which is divided between travelling and harvesting. If the harvesting period were fixed, the agent may find it optimal to wait, i.e., to let the resource grow before harvesting, which occurs if the harvesting period is, in 
this sense, too long. Here, however, the travelling period gives the agent the possibility to adjust the length of the harvesting period by choosing the arrival time. In the case of exponential growth, the agent adjusts the travelling time so as to avoid any waiting period before starting to harvest. A similar finding holds in the case of logistic growth; yet, if the harvesting capacity is sufficiently high, it becomes optimal for the agent to employ a three-part harvesting strategy: In the beginning and at the end of the harvesting period the agent harvests at the maximum capacity, but reduces harvesting in an intermediate time interval, during which the agent harvests the sustainable level by equalising the harvesting rate and the growth rate of the stock. The length of this intermediate time interval with sustainable harvesting increases in the harvesting capacity. In fact, this sustainable harvesting level also represents the optimal steady state for an infinite time horizon. Yet, as the planning horizon of the agent is finite, the agent eventually abandons the sustainable policy and resumes harvesting at the maximal level until the end of the planning period. In sum, irrespective of the specification of the growth function, the agent accomplishes to economise on the travelling costs while choosing an optimal harvesting policy that involves periods of maximal harvesting but may also include a sustainable, well balanced interim harvesting period.

Further robustness checks (Appendix A) that include the case of a positive discount rate and bounds on acceleration show that those insights continue to hold in a more general framework. The major modifications, though, are these: A positive discount rate lets the agent postpone part of the travelling cost by shifting the acceleration profile towards the future. A binding lower bound on acceleration inhibits this cost-shifting effect as the agent is required to slow down earlier, and thus has to accelerate earlier.

In sum, we have demonstrated that the acknowledgement of the spatial dimension in the classical problem of managing a renewable resource leads to interesting and economically relevant, yet analytically tractable results. Our set-up allows for the introduction of further realistic features, extending the theory to the case where the agent faces a transportation problem that is temporarily and spatially linked to a resource-gathering problem.

Acknowledgments We are grateful to Peter M. Kort, Gustav Feichtinger, Florian O. O. Wagener, and the participants of the International Conference Optimal Control and Differential Games dedicated to the 110th anniversary of L.S. Pontryagin for valuable comments and suggestions. Also, we would like to thank two anonymous referees for their very helpful and detailed comments. Thorsten Upmann acknowledges funding by HIFMB, a collaboration between the Alfred-Wegener-Institute, Helmholtz-Center for Polar and Marine Research, and the Carl-von-Ossietzky University Oldenburg, initially funded by the Ministry for Science and Culture of Lower Saxony (MWK) and the Volkswagen Foundation through the "Niedersächsisches Vorab" grant program (grant number ZN3285).
Open Access This article is licensed under a Creative Commons Attribution 4.0 International License, which permits use, sharing, adaptation, distribution and reproduction in any medium or format, as long as you give appropriate credit to the original author(s) and the source, provide a link to the Creative Commons licence, and indicate if changes were made. The images or other third party material in this article are included in the article's Creative Commons licence, unless indicated otherwise in a credit line to the material. If material is not included in the article's Creative Commons licence and your intended use is not permitted by statutory regulation or exceeds the permitted use, you will need to obtain permission directly from the copyright holder. To view a copy of this licence, visit http:// creativecommonshorg/licenses/by/4.0/.

\section{Appendix A: Robustness analysis: a positive discount rate and bounds on acceleration}

We here explore how a positive discount rate and bounds on acceleration affect the optimal travelling policy. We maintain our specification of travelling cost (15). Then, with a positive discount rate, the objective function thus equals ${ }^{18}$

$J_{1}\left(a(\cdot), t_{1}\right)=\int_{0}^{t_{1}} e^{-\rho t}\left(c v(t)+a(t)^{2}\right) \mathrm{d} t$.

Acknowledging the law of motion (1a), the Hamiltonian is given by

$\mathcal{H}_{1}=-c v(t)-a(t)^{2}+\psi_{1}(t) v(t)+\psi_{2}(t) a(t)$.

We assume that acceleration is bounded to $\mathscr{A}=[\underline{a}, \bar{a}]=$ $[-1,+1]$, and the corresponding Lagrangean reflecting this constraint equals

$\mathcal{L}=\mathcal{H}_{1}+\lambda_{1}(t)(1+a(t))+\lambda_{2}(t)(1-a(t))$.

Then, the necessary conditions for the optimal solution are given by

$$
\begin{aligned}
a(t) & =\frac{1}{2}\left(\pi_{2}(t)+\lambda_{1}(t)-\lambda_{2}(t)\right), \\
\dot{x}(t) & =v(t), \quad x(0)=0, \quad x\left(t_{1}\right)=x_{1}, \\
\dot{v}(t) & =a(t), \quad v(0)=0, \quad v\left(t_{1}\right)=0, \\
\dot{\pi}_{1}(t) & =-\frac{\partial \mathscr{L}}{\partial x(t)}+\rho \pi_{1}(t)=\rho \pi_{1}(t), \\
\dot{\pi}_{2}(t) & =-\frac{\partial \mathscr{L}}{\partial v(t)}+\rho \pi_{2}(t)=-\pi_{1}(t)+\rho \pi_{2}(t)+c . \text { (A.1e) }
\end{aligned}
$$

Using the yet unspecified initial values $\pi_{1}(0)=m_{1}$ and $\pi_{2}(0)=c_{1}$, the latter two equations yield

$\pi_{1}(t)=m_{1} e^{\rho t}, \quad \pi_{2}(t)=e^{\rho t}\left(c_{1}-m_{1} t\right)+\frac{c}{\rho}\left(e^{\rho t}-1\right)$.

\footnotetext{
${ }^{18}$ In order to avoid that a negative speed, i.e., moving backwards, reduces cost, it is preferable to write $c|v|$ instead of $c v$. However, we will assume that $c$ is sufficiently low so that moving backwards is never optimal and both cost components are non-negative along the optimal path. Still, for sufficiently high values of $c$, moving backwards may be part of the solution unless we replace $c v$ by $c|v|$.
} 
To be able to clearly identify the effects of the bounds on the controls, we first solve, as a point of reference, the problem when $a$ is unbounded.

\section{A.1 Analysis of the unbounded solution}

In this case we have $\lambda_{1}(t)=0=\lambda_{2}(t)$, so that Eq. (A.1a) reduces to $a(t)=\pi_{2}(t) / 2$. Substituting this into Eq. (A.1b) and Eq. (A.1c), we obtain

$\dot{x}(t)=v(t), \quad \dot{v}(t)=\frac{\pi_{2}(t)}{2}$.

Then, substituting Eq. (A.2) into Eq. (A.3) yields the system

$$
\begin{aligned}
\dot{x}(t)=v(t), \quad \dot{v}(t) & =\frac{1}{2}\left(e^{\rho t}\left(c_{1}-m_{1} t\right)+\frac{c}{\rho}\left(e^{\rho t}-1\right)\right), \\
x(0) & =0, \quad v(0)=0,
\end{aligned}
$$

the solution of which is given by

$$
\begin{aligned}
x(t)= & \frac{1}{4 \rho^{3}}\left[c\left(2 e^{\rho t}-2-\rho t(\rho t+2)\right)-2 c_{1} \rho\left(\rho t-e^{\rho t}+1\right)\right. \\
& \left.-2 m_{1}\left(\rho t+e^{\rho t}(\rho t-2)+2\right)\right], \\
v(t)= & \frac{1}{2 \rho^{2}}\left[c\left(e^{\rho t}-1-\rho t\right)+e^{\rho t}\left(c_{1} \rho+m_{1}-m_{1} \rho t\right)\right. \\
& \left.-c_{1} \rho-m_{1}\right] .
\end{aligned}
$$

Finally, using the terminal conditions $x\left(t_{1}\right)=x_{1}$ and $v\left(t_{1}\right)=0$, we obtain the constants $c_{1}$ and $m_{1}$. Applying those in Eq. (A.2), and using that result to calculate $a(t)=$ $\pi_{2}(t) / 2$, we obtain a generalisation of Proposition 6, with the limiting case $\rho \rightarrow 0$ recouping our former result:

Proposition 10 Given arrival time $t_{1}$, the optimal travelling policy is given by

$a(t)=\frac{1}{4 \rho} \frac{\theta_{1}\left(t, t_{1}\right)}{\theta_{3}\left(t_{1}\right)} c-\frac{\theta_{2}\left(t, t_{1}\right)}{\theta_{3}\left(t_{1}\right)} x_{1}$,

and the associated travelling cost amounts to

$J_{1}^{*}\left(t_{1}\right)=-\frac{1}{\kappa_{0}\left(t_{1}\right)}\left(\kappa_{1}\left(t_{1}, x_{1}\right)+\kappa_{2}\left(t_{1}, x_{1}\right) c+\kappa_{3}\left(t_{1}\right) c^{2}\right)$,

$$
\begin{aligned}
& \text { where } \\
& \qquad \begin{aligned}
\theta_{1}\left(t, t_{1}\right) \equiv & 2+2 e^{2 \rho t_{1}}-2 e^{\rho t_{1}}\left(\rho^{2} t_{1}^{2}+2\right) \\
& +\rho t_{1} e^{\rho t}\left(\rho t\left(2+\rho t_{1}\right)+\rho t_{1}+4\right) \\
& -\rho t_{1} e^{\rho\left(t+t_{1}\right)}\left(\rho t\left(2-\rho t_{1}\right)+\rho t_{1}\left(\rho t_{1}-3\right)+4\right), \\
\theta_{2}\left(t, t_{1}\right) \equiv & \rho^{2} e^{\rho t}\left(1+\rho t+e^{\rho t_{1}}\left(\rho\left(t_{1}-t\right)-1\right)\right), \\
\theta_{3}\left(t_{1}\right) \equiv & e^{\rho t_{1}}\left(\rho^{2} t_{1}^{2}+2\right)-e^{2 \rho t_{1}}-1, \\
\kappa_{0}\left(t_{1}\right) \equiv & 2+\rho^{2} t_{1}^{2}-2 \cosh \left(\rho t_{1}\right), \\
\kappa_{1}\left(t_{1}, x_{1}\right) \equiv & \rho^{3} x_{1}^{2} e^{-\rho t_{1}}\left(e^{\rho t_{1}}-1\right), \\
\kappa_{2}\left(t_{1}, x_{1}\right) \equiv & 8 \rho^{4} t_{1} x_{1}\left(\rho t_{1} \cosh \left(\rho t_{1} / 2\right)-2 \sinh \left(\rho t_{1} / 2\right)\right), \\
\kappa_{3}\left(t_{1}\right) \equiv & \sinh \left(\rho t_{1} / 2\right)\left(\rho^{4} t_{1}^{4}+12 \rho^{2} t_{1}^{2}-8 \cosh \left(\rho t_{1}\right)+8\right) \\
& -4 \rho^{3} t_{1}^{3} \cosh \left(\rho t_{1} / 2\right) .
\end{aligned}
\end{aligned}
$$

Proof The result follows from the preceding analysis.

We thus find that for a fixed arrival time $t_{1}$, the optimal control $a$ is an affine function of $c$ (as are $x, v$ and $\pi_{2}$, while $\pi_{1}$ is independent of $c$ ); and for this reason, the minimised travelling cost $J_{1}^{*}$ is a polynomial of order 2 of $c$.

\section{A.2 Analysis of the bounded solution}

We now take into account that the control is actually bounded to $a(t) \in \mathscr{A}$. The optimal control of the unbounded case may either hit the upper bound or the lower bound, or both. For illustrative purposes, let us henceforth assume that the parameters are chosen such that the lower bound becomes binding, while the upper bound does not. In this case, it suffices to consider the constraint $a \geq \underline{a}$ only. (The case $a \leq \bar{a}$ can be analysed in an analogous way.)

Assume that for the unbounded solution, the lower bound becomes binding at time $\underline{t} \in\left(0, t_{1}\right)$. We refer to $\underline{t}$ as the hitting time. The optimal hitting time for the bounded case $\xi$, though, has to be determined as part of the solution of the bounded problem. We know that we have $a(t)=\underline{a}=-1$ for all $t$ in the final interval $\left(\xi, t_{1}\right]$. Consequently, we must have $\xi<\underline{t}$, for if $\xi=\underline{t}$ the remaining time would only suffice to guarantee the terminal condition $v\left(t_{1}\right)=0$, if we were able to set $a<\underline{a}$. Thus, during the final time interval $\left(\xi, t_{1}\right]$, the solution must satisfy

$\dot{x}(t)=v(t), \quad \dot{v}(t)=-1, \quad x\left(t_{1}\right)=x_{1}, \quad v\left(t_{1}\right)=0$,

the solution of which is

$x(t)=\frac{1}{2}\left(-\left(t-t_{1}\right)^{2}+2 x_{1}\right), \quad v(t)=t_{1}-t$.

In particular, these equations must be satisfied at the optimal hitting time $\xi$, and thus they provide the right-hand boundary conditions for the time interval $[0, \xi]$. Hence, we obtain a system consisting of Eqs. (A.1d), (A.1e), (A.3) and the boundary conditions:

$$
\begin{aligned}
x(0)=0, \quad v(0)=0, \quad x(\xi) & =\frac{1}{2}\left(-\xi^{2}+2 \xi t_{1}-t_{1}^{2}+2 x_{1}\right), \\
v(\xi) & =t_{1}-\xi .
\end{aligned}
$$

The solution of this system yields the optimal travelling policy for the interval $[0, \xi]$, which is again an affine function of $c$. Hence, putting the pieces together, we find:

Proposition 11 Given arrival time $t_{1}$ and the constraint $a \in \mathscr{A} \equiv[-1,1]$, the optimal travelling policy is given by

$a(t, \xi)= \begin{cases}\frac{1}{4 \rho} \frac{\theta_{1}(t, \xi)}{\theta_{3}(\xi)} c+\frac{\rho}{2} \frac{\theta_{5}(t, \xi)}{\theta_{3}(\xi)}, & \forall t \in[0, \xi] \\ -1, & \forall t \in\left(\xi, t_{1}\right]\end{cases}$ 
where the optimal hitting time $\xi$ is determined by $a(\xi, \xi)=$ $-1, \theta_{1}$ and $\theta_{3}$ are as defined in Proposition 10, and

$$
\begin{aligned}
\theta_{5}(t, \xi) \equiv & e^{\rho t}\left[-4 \xi+\rho^{2} t\left(-\xi^{2}+t_{1}^{2}-2 x_{1}\right)\right. \\
& \left.+\rho\left(t_{1}-\xi\right)\left(\xi+2 t+t_{1}\right)+4 t_{1}-2 \rho x_{1}\right] \\
& +e^{\rho(\xi+t)}\left[4 \xi-\rho\left(t_{1}-\xi\right)\left(-3 \xi+2 t+t_{1}\right)\right. \\
& \left.+\rho^{2}(\xi-t)\left(\left(t_{1}-\xi\right)^{2}-2 x_{1}\right)-4 t_{1}+2 \rho x_{1}\right] .
\end{aligned}
$$

Proof The result follows from the preceding analysis.

The presence of both cost-shifting effects can formally be shown:

Corollary 3 Given arrival time $t_{1}$ and the constraint $a \in$ $\mathscr{A} \equiv[-1,1]$, and the associated optimal travelling policy, we have

(i) $\partial J_{1}^{*} / \partial \rho<0$ for all $\rho$ and $c \leq \bar{c}(\rho)$, where $\bar{c}(\rho)$ is the highest cost parameter such that for any $c>\bar{c}(\rho)$ the agent begins the travelling period with moving backwards, i.e., $\left.a(0)\right|_{c=\bar{c}(\rho)}=0$, (see also fn. 18);

(ii) $\partial a(0, \xi) / \partial c<0$, i.e., the acceleration at the beginning of the period falls if the cost of speed increases.

Proof (i): The result follows from the Envelope Theorem. (ii): Using Proposition 11, and evaluating the optimal control at $t=0$, we obtain (with slight abuse of notation):

$$
\begin{aligned}
\frac{\partial a(0, \xi)}{\partial c} & =\frac{1}{2 \rho}\left(-1+\gamma\left(\rho t_{1}\right)\right) \quad \text { where } \\
\gamma(z) & \equiv \frac{z\left(e^{z} z^{2}-3 e^{z} z-z+4 e^{z}-4\right)}{2\left(-e^{z} z^{2}-2 e^{z}+e^{2 z}+1\right)} .
\end{aligned}
$$

Using l'Hôpital's rule, we find $\lim _{z \rightarrow 0} \gamma(z)=1$ and $\lim _{z \rightarrow \infty} \gamma(z)=0$. Moreover, it is tedious but straightforward to show that $\gamma^{\prime}<0$, and hence we have $\partial a(0, \xi) / \partial c<0$.

\section{Appendix B: Proofs}

\section{Proof of Lemma 1}

Proof Assume, on the contrary, that $\pi\left(t_{1}\right)>M$. Since $s\left(t_{1}\right)=2$, it follows from Eq. (9c) that $\dot{\pi}\left(t_{1}\right)=-h\left(t_{1}\right) M+$ $\pi\left(t_{1}\right)\left(2+h\left(t_{1}\right)\right)>0$. Since $h(t)=0$ as long as $\pi(t)>M$, the stock remains at its starting value $s\left(t_{1}\right)=2$. Given this, there is no turning point in the evolution of $\pi$, and thus $\pi$ continues to grow, i.e., we have $\dot{\pi}(t)>0$ for all $t$. Yet, this contradicts the transversality condition $\pi(T)=0$ and thus proves our claim $\pi\left(t_{1}\right)<M$, and thus $h\left(t_{1}\right)=\bar{h}$.

\section{Proof of Lemma 2}

Proof From Eq. (12a) we can calculate the critical time horizon $T_{c}$ for which at some point in time $t_{c}$ the trajectory goes through the point $\left(s\left(t_{c}\right), \pi\left(t_{c}\right)\right)=(1, M)$. Using that information and evaluating $\pi$ at $T_{c}$ yields $t_{c}=t_{1}+$ $\frac{1}{2-\bar{h}} \log \left(\frac{\bar{h}}{2(\bar{h}-1)}\right)$ and thus Eq. (11).

\section{Proof of Lemma 3}

Proof The derivative of the value function $J_{2 A}^{*}\left(t_{1}\right)$ is given by:

$\frac{\mathrm{d} J_{2 A}^{*}\left(t_{1}\right)}{\mathrm{d} t_{1}}=-s_{0} M e^{t_{1}} \frac{\bar{h}}{\bar{h}-1}\left(\bar{h} e^{(\bar{h}-1)\left(t_{1}-T\right)}-1\right)$.

It is then straightforward to show that, irrespective of the sign of $\bar{h}-1$, the sign of the derivative of $J_{2 A}^{*}$ depends on whether the switching point $\tau$ is before or after the arrival time $t_{1}$, and hence Eq. (23) follows.

\section{Proof of Proposition 3}

Proof (A similar proof can be found in Hocking 1991.) Since the Hamiltonian is autonomous, it is constant along the optimal trajectory (see, for example, Intriligator 1971), p. 350-355). We can therefore characterise the trajectories in the $(s, \pi)$ plane for $h=0$ and for $h=\bar{h}$. Let $K$ denote the level of the Hamiltonian, then the optimal trajectories are characterised by the equations

$\pi(t)=\frac{K}{2 s(t)-s^{2}(t)} \quad$ and $\quad \pi(t)=\frac{K-s(t) \bar{h}}{2 s(t)-s^{2}(t)-s(t) \bar{h}}$

for $h=0$ and $h=\bar{h}$, respectively. The $h=0$ trajectories have their minima at $s=1$, and the trajectories with $h=\bar{h}$ attain their maxima along the curve

$\pi(t)=\frac{-\bar{h}}{2-2 s(t)-\bar{h}} \quad$ for $s>1-\frac{1}{2} \bar{h}$.

Both types of trajectories are depicted in Fig. 5 for a low (left diagram) and a high (right diagram) harvesting capacity. The trajectories starting from $s\left(t_{1}\right)=2$ reach the horizontal axis at time $T$, i.e., $\pi(T)=0$. Those trajectories with $\bar{h}<1$ cross the horizontal axis at a point to the right of $2-\bar{h}$, that is $s(T)>2-\bar{h}$. If $\bar{h}$ is sufficiently small, the trajectory does not reach the $\pi=M$ line (for $M=1$ see Fig. 5, left). Since the locus of maxima crosses the point $(1, M)$, the critical trajectory is that one which achieves its maximum at this point (see Fig. 5, right). Because the trajectories do not cross the horizontal axis to the left of $2-\bar{h}$, the critical trajectory must feature $\bar{h}>1$. It thus follows that the critical harvesting capacity exceeds unity, $\bar{h}_{c}>1$. 


\section{Proof of Proposition 5}

Proof That Eq. (14a) is indeed the optimal harvesting policy can be seen by noting that $\pi=M$ is a singular level. Since we already know that $\pi\left(t_{1}\right)<M$, it follows that $\dot{\pi} \geq 0$ at the time the singular level $\pi=M$ is reached. If we have $\pi=M$ for some time interval with positive length, then $\dot{\pi}=0$ and hence we must have $s=1 \Rightarrow \dot{s}=0 \Rightarrow h=1$ from Eq. (9a).

After completing the singular path we cannot have a path with $h=0$. This can be seen as follows: $h=0$ implies $\dot{s}>0$, which in turn implies that, because $s=1$ on the singular arc, $s>1$ right after the singular arc. Jointly with $h=0$ this in turn implies that $\dot{\pi}>0$. Hence, we enter a path where both $s$ and $\pi$ are growing so that the transversality condition $\pi(T)=0$ cannot be satisfied. We thus conclude that the optimal policy must proceed with $h=\bar{h}$ after completing the singular path-and thus the policy in Eq. (14a) is optimal.

The total length of the harvesting sub-periods $\left[t_{1}, t_{2}\right)$ and $\left[t_{3}, T\right]$ amounts to $T_{c}-t_{1}$ and is thus given by Eq. (11). Therefore harvesting during these sub-periods brings about the same profit as in Case A, i.e., $J_{2 A}^{c}$ given by Eq. (13); while during the time interval $\left[t_{2}, t_{3}\right)$ the resulting profit equals $h=1$ times the length of the harvesting period: $t_{3}-t_{2}=T-T_{c}$. So, we obtain $J_{2 B}^{*}=J_{2 A}^{c}+M \int_{t_{2}}^{t_{3}} 1 \mathrm{~d} t=$ $J_{2 A}^{c}+M\left(T-T_{c}\right)$. Finally, substituting the definition of $T_{c}$, Eq. (11), yields Eq. (14b).

\section{Proof of Proposition 7}

Proof Using the maximised Hamiltonian of Section "Fixed travelling period", $\mathcal{H}_{1}^{*}=36 x_{1}^{2} / t_{1}^{4}$, the transversality condition Eq. (20) gives Eq. (22b). Since $\mathcal{H}_{1}^{*}=$ $-\mathrm{d} J_{1}^{*} / \mathrm{d} t_{1}>0$, the derivative $\mathrm{d} J_{2 A}^{*}\left(t_{1}\right) / \mathrm{d} t_{1}$ must be negative in order for Eq. (22b) to have a solution $t_{1}^{*}$. By Lemma 3, $\mathrm{d} J_{2 A}^{*}\left(t_{1}\right) / \mathrm{d} t_{1}$ is negative if, and only if, the switching point $\tau$ is before the arrival time: $t_{1}^{*}>T-\delta \equiv \tau$, implying that Case A applies.

\section{References}

Ahn K, Rakha H, Trani A, Van Aerde M (2002) Estimating vehicle fuel consumption and emissions based on instantaneous speed and acceleration levels. Journal of Transportation Engineering-ASCE 128(2):182-190

Amit R (1986) Petroleum reservoir exploitation: Switching from primary to secondary recovery. Oper Res 34(4):534-549

Aniţa S., Behringer S, Moşneagua A.-M., Upmann T (2019) Optimal harvesting of a spatially distributed renewable resource with endogenous pricing. Mathematical Modelling of Natural Phenomena 14(1:101):1-13

Behringer S, Upmann T (2014) Optimal harvesting of a spatial renewable resource. J Econ Dyn Control 42:105-120
Belyakov AO, Davydov AA, Veliov VM (2015) Optimal cyclic exploitation of a renewable resources. J Dyn Control Syst 21(3):475-494

Belyakov AO, Davydov AA, Veliov VM (2017) (2017). Optimal Cyclic harvesting of renewable resource. Doklady mathematics 96(2), 472-474. Published in Russian in Doklady Akademii Nauk 476(4):371-374

Belyakov AO, Veliov VM (2014) Constant versus periodic fishing: Age structured optimal control approach. Mathematical Modelling of Natural Phenomena 9(4):20-37

Bertolazzi E, Frego M (2018) Semianalytical minimum-time solution for the optimal control of a vehicle subject to limited acceleration. Optimal Control Applications and Methods 39(2, SI):774-791

Bifulco GN, Galante F, Pariota L, Spena MR (2015) A linear model for the estimation of fuel consumption and the impact evaluation of advanced driving assistance systems. Sustainability 7(10):1432614343

Boucekkine R, Saglam C, Vallee T (2004) Technology adoption under embodiment: a Two-Stage optimal control approach. Macroecon Dyn 8(2):250-271

Brock WA, Xepapadeas A (2008) Diffusion-induced Instability and Pattern Formation in Infinite Horizon Recursive Optimal Control. J Econ Dyn Control 32(9):2745-2787

Brock WA, Xepapadeas A (2010) Pattern Formation, Spatial Externalities and Regulation in Coupled Economic-ecological Systems. J Environ Econ Manag 59(2):149-164

Caulkins JP, Feichtinger G, Grass D, Hartl RF, Kort PM, Seidl A (2013) When to make proprietary software open source. J Econ Dyn Control 37(6):1182-1194

Chu W.-L. (2017) Strategies to enhance production of microalgal biomass and lipids for biofuel feedstock. Eur J Phycol 52(4):419437

Clark CW (2010) Mathematical Bioeconomics, 3rd ed. John Wiley \& Sons, New Jersey

Conrad JM, Clark CW (1987) Natural resource economics: Notes and problems. Cambridge University Press, Cambridge

Conrad JM, Smith MD (2012) Nonspatial and spatial models in bioeconomics. Nat Resour Model 25(1):52-92

Da Lara M, Doyen L (2008) Sustainable managment of natural resources: Mathematical models and methods. Springer, Berlin

Ding W, Lenhart S (2009) Optimal harvesting of a spatially explicit fishery model. Nat Resour Model 22(2):173-211

Fan M, Wang K (1998) Optimal harvesting policy for single population with periodic coefficients. Math Biosci 152(2):165-177

Feichtinger G, Tragler G, Veliov VM (2003) Optimality conditions for Age-Structured control systems. J Math Anal Appl 288(1):47-68

Gordon HS (1954) The economic theory of a Common-Property resource: the fishery. J Polit Econ 62(2):124-142

Grass D, Hartl RF, Kort PM (2012) Capital accumulation and embodied technological progress. J Optim Theory Appl 154(2):588-614

Grass D, Uecker H, Upmann T (2019) Optimal fishery with coastal catch. Nat Resour Model 32(32):e12235

Hocking LM (1991) Optimal Control: An introduction to the theory with applications. Oxford University Press, New Jersey

Hritonenko N, Yatsenko Y (2006) Optimization of harvesting return from Age-Structured population. J Bioecon 8(2):167-179

Hull DG (2003) Optimal control theory for applications. Mechanical engineering series. Springer, Berlin

Intriligator MD (1971) Mathematical optimization and economic theory (reprint 2002 ed.), Volume 39 of classics in applied mathematics. SIAM: Society for industrial and applied mathematics

Kamien MI, Schwartz NL (1991) Dynamic Optimization: The Calculus of Variations and Optimal Control in Economics and Management (2nd ed.), Volume 31 of Advanced Textbooks in Economics. Amsterdam. Elsevier, North-Holland 
Kelly MRJ, Yulong X, Lenhart S (2016) Optimal fish harvesting for a population modeled by a nonlinear parabolic partial differential equation. Nat Resour Model 29(1):36-70

Krawczyk JB, Serea O.-S. (2013) When can it be not optimal to adopt a new technology? A viability theory solution to a two-stage optimal control problem of new technology adoption. Optimal Control Applications and Methods 34(2): 127-144

Kroetz K, Sanchirico JN (2015) The bioeconomics of Spatial-Dynamic systems in natural resource management. Ann Rev Resour Econ 7(1):189-207

Liski M, Kort PM, Novak A (2001) Increasing returns and cycles in fishing. Resour Energy Econ 23(3):241-258

Léonard D, Long NV (1992) Optimal control theory and static optimization in economics. Cambridge University Press, Cambridge

Long NV, Prieur F, Tidball M, Puzon K (2017) Piecewise ClosedLoop equilibria in differential games with regime switching strategies. J Econ Dyn Control 76:264-284

Makris M (2001) Necessary conditions for infinite-horizon discounted two-stage optimal control problems. J Econ Dyn Control 25(12):1935-1950

Moeller HV, Neubert MG (2013) Habitat damage, marine reserves, and the value of spatial management. Ecol Appl 23(5):959-971

Montero JA (2000) A uniqueness result for an optimal control problem on a diffusive elliptic Volterra-Lotka type equation. J Math Anal Appl 243(1):13-31

Montero JA (2001) A study of the profitability for an optimal control problem when the size of the domain changes. Nat Resour Model 14(1):139-146

Moser E, Seidl A, Feichtinger G (2014) History-dependence in production-pollution-trade-off models: A multi-stage approach. Ann Oper Res 222(1):457-481

Neubert MG (2003) Marine reserves and optimal harvesting. Ecol Lett 6(9):843-849

Neubert MG, Herrera GE (2008) Triple benefits from spatial resource management. Theoretical Ecology 1(1):5-12

Pindyck RS (1984) Uncertainty in the theory of renewable resource markets. Rev Econ Stud 51(2):289-303

Polasky S, de Zeeuw A, Wagener F (2011) Optimal management with potential regime shifts. J Environ Econ Manag 62(2):229-240

Pontryagin LS, Boltyanskii VG, Gamkrelidze RV, Mishchenko EF (1962) The Mathematical Theory of Optimal Processes. New York, London: Interscience Publishers John Wiley \& Sons. Inc. English translation by K. N. Trirogoff

Robinson EJZ, Albers HJ, Williams JC (2008) Spatial and temporal modeling of community non-timber forest extraction. J Environ Econ Manag 56(3):234-245

Robinson EJZ, Williams JC, Albers HJ (2002) The influence of markets and policy on spatial patterns of non-timber forest product extraction. Land Econ 78(2):260-271

Saglam C (2011) Optimal pattern of technology adoptions under embodiment: a Multi-Stage optimal control approach. Optimal Control Applications and Methods 32(5):574-586

Sanchirico JN, Wilen JE (1999) Bioeconomics of spatial exploitation in a patchy environment. J Environ Econ Manag 37(2):129-150
Schaefer MB (1954) Some aspects of the dynamics of populations important to the management of the commercial marine fisheries. Bulletin of Inter-American Tropical Tuna Commission 1(2):25-56

Seidl A, Caulkins JP, Hartl RF, Kort PM (2018) Serious strategy for the makers of fun: analyzing the option to switch from pay-to-play to free-to-play in a two-stage optimal control model with quadratic costs. Eur J Oper Res 267(2):700-715

Shurin JB, Abbott RL, Deal MS, Kwan GT, Litchman E, McBride RC, Mandal S, Smith VH (2013) Industrial-strength ecology: Trade-offs and opportunities in algal biofuel production. Ecol Lett 16(11):1393-1404

Sirén A., Parvinen K (2015) A spatial bioeconomic model of the harvest of wild plants and animals. Ecol Econ 116:201-210

Skonhoft A, Vestergaard N, Quaas M (2012) Optimal harvest in an age structured model with different fishing selectivity. Environ Resour Econ 51(4):525-544

Smith VL (1968) Economics of production from natural resources. Am Econ Rev 58(3):409-431

Tahvonen O (2008) Harvesting an age-structured population as biomass: Does it work? Nat Resour Model 21(4):525-550

Tahvonen O (2009a) Economics of harvesting Age-Structured fish populations. J Environ Econ Manag 58(3):281-299

Tahvonen O (2009b) Optimal harvesting of Age-Structured fish populations. Mar Resour Econ 24(2):147-169

Tahvonen O, Quaas MF, Schmidt JO, Voss R (2013) Optimal harvesting of an age-structured schooling fishery. Environmental and Resource Economics 54(1):21-39

Thieme HR (2003) Mathematics in Population Biology. Princeton University Press, Princeton and Oxford

Tomiyama K (1985) Two-stage optimal control problems and optimality conditions. J Econ Dyn Control 9(3):317-337

Tomiyama K, Rossana RJ (1989) Two-stage optimal control problems with an explicit switch point dependence: Optimality criteria and and example of delivery lags and investment. J Econ Dyn Control 13(3):319-337

Ummalyma SB, Gnansounou E, Sukumaran RK, Sindhu R, Pandey A, Sahoo D (2017) Bioflocculation: an alternative strategy for harvesting of microalgae - an overview. Bioresour Technol 242(SI):227-235

Wörz S, Bernhardt H (2017) A novel method for optimal fuel consumption estimation and planning for transportation systems. Energy 120:565-572

Yoshioka H, Yaegashi Y (2018) Singular stochastic control model for algae growth management in dam downstream. J Biol Dyn 12(1):242-270

Zelikin MI, Lokutsievskii LV, Ckopintsev SV (2017) On optimal harvesting of a resource on a circle. Mathematical notes 102(4):521532. Published in Russian language in Matematicheskie Zametki, 2017

Zhu L, Nugroho YK, Shakeel SR, Li Z, Martinkauppi B, Hiltunen E (2017) Using microalgae to produce liquid transportation biodiesel: What is next? Renewable \& Sustainable Energy Reviews 78:391-400 